Packetized predictive control of stochastic systems over bit-rate limited channels with packet loss

by Daniel E. Quevedo, Jan Østergaard \& Dragan Nešić

Copyright (C) 2011 IEEE.

This is an author-prepared version of the article, reprinted from IEEE Transactions on Automatic Control, Vol. 56, Issue 12, p. 2854-2868.

http://dx.doi.org/10.1109/TAC.2011.2139410

This material is posted here with permission of the IEEE. Such permission of the IEEE does not in any way imply IEEE endorsement of any of University of Newcastle's products or services. Internal or personal use of this material is permitted. However, permission to reprint/republish this material for advertising or promotional purposes or for creating new collective works for resale or redistribution must be obtained from the IEEE by writing to pubs-permissions@ieee.org. By choosing to view this document, you agree to all provisions of the copyright laws protecting it. 


\title{
Packetized Predictive Control of Stochastic Systems over Bit-Rate Limited Channels with Packet Loss
}

\author{
Daniel E. Quevedo, Member, IEEE, Jan Østergaard, Member, IEEE, \\ and Dragan Nešić, Fellow, IEEE
}

\begin{abstract}
We study a control architecture for linear timeinvariant plants with random disturbances and where a network is placed between the controller output and the plant input. The network imposes a constraint on the expected bit-rate and is affected by random i.i.d. dropouts. Dropout-rates and acknowledgments of receipt are not available at the controller side. To achieve robustness with respect to i.i.d. dropouts, the controller transmits data packets containing quantized plant input predictions. These are provided by an appropriate optimal entropy coded dithered lattice vector quantizer. Within this context, we derive stochastic stability results and provide a noiseshaping model of the closed loop system. This model is employed for performance analysis by using rate-distortion theory.
\end{abstract}

\section{INTRODUCTION}

The interest in closed loop control over communication networks has increased tremendously in recent years; see, e.g., [1]-[4] and the many references therein. In particular, Ethernet in its wired (hub-based and switched) and wireless forms (IEEE 802.11) is increasingly being adopted as a low level control network technology, see [5], [6]. The reasons for this move towards Networked Control Systems (NCS's) are manifold, including lower cost, higher reliability, interoperability of devices, and easier installation and maintenance.

From a control design perspective, many interesting challenges are associated with NCS's. For example, due to the inherent bit-rate limitations associated with digital networks, signals need to be quantized and coded prior to transmission; see, e.g., [2], [7], [8]. Furthermore, the network may induce random delays and data-dropouts; see, e.g., [1], [9], [10]. It turns out that, in a NCS, links are not transparent, often constituting a significant bottleneck in the achievable performance. Thus, successful NCS design methods need to consider both control and communication aspects.

For the design of NCS's one can often take advantage of the fact that in contemporary communication networks, data

Manuscript received September 15, 2009; revised August 25, 2010. This paper was presented in part at CDC'09. Recommended by Associate Editor K. H. Johansson. This research was supported under Australian Research Council's Discovery Projects funding scheme (project numbers DP0988601 and DP1094326) and Future Fellowship FT0990727. The work of J. Østergaard is supported by the Danish Research Council for Technology and Production Sciences, grant no. 274-07-0383.

Daniel Quevedo is with the School of Electrical Engineering \& Computer Science, The University of Newcastle, Callaghan, NSW 2308, Australia; e-mail: dquevedo@ieee.org. Jan Østergaard is with the Department of Electronic Systems, Aalborg University, Denmark; e-mail: janoe@ieee.org. Dragan Nešić is with the Department of Electrical and Electronic Engineering, The University of Melbourne, Carlton, VIC 3010, Australia; Email: dnesic@unimelb.edu.au. is sent in large time-stamped packets. ${ }^{1}$ This motivates the development of control methods in which packets of signal predictions are sent. These are calculated at the transmission side to compensate for random time delays and packetdropouts. At the receiver node, packets are buffered and only the latest relevant value is used. In particular, the idea of sending packets with plant input predictions obtained via a predictive controller (hereafter termed packetized predictive controller (PPC)) was proposed in [11] for the teleoperation of prestabilized constrained nonlinear plants. The concept also underlies more recent NCS configurations described, e.g., in [12]-[19]. Experimental results of NCS's which use PPCs are promising. However, only limited results on theoretic aspects exist, specially for the case of NCS's with disturbances, see [12], [16], [20], [21]. In particular, in [20] we have shown that, under suitable assumptions, the optimal cost provided by the PPC optimizations at successful transmission instants can be designed to constitute an ISS-Lyapunov function for the NCS.

Since with PPCs each data-packet contains a possibly large number of plant input predictions, even if high packet bit-rates are allowed, quantization effects may become important. Nevertheless, no works on PPCs have treated bit-rate limitations or quantization issues. One of the purposes of the present work is to elucidate the trade-off between bit-rates and number of predictions in each packet.

The present paper examines a NCS for discrete-time linear time invariant plants with random disturbances. Signal transmission is over a bit-rate limited network, which is affected by random packet-dropouts and does not provide acknowledgments of successful transmission, see Fig. 1. To be amenable to practical situations where dropout-rates are unknown, the PPC studied in the present work is designed without requiring knowledge of the packet dropout distribution. ${ }^{2}$ The controller explicitly incorporates bit-rate constraints of the network by sending quantized finite-horizon plant input sequences. These are provided by an appropriate entropy constrained lattice vector quantizer. For Bernoulli dropouts, we combine results from Markov Jump-Linear Systems, see, e.g., [25], [26] and stability results for PPC presented in our recent work [20], to develop sufficient conditions for stationarity and mean-square stability of the NCS. We also provide analytical expressions for the spectral densities of the signals of interest. This spectral

\footnotetext{
${ }^{1}$ For example, the frame format in IP based Ethernet allows for a datapacket of 46-1500 bytes, the overhead being 26 bytes. Time-stamping can be easily carried out, e.g., by invoking the Real Time Transport Protocol.

${ }^{2}$ This stands in contrast to other works, e.g., [22]-[24], where controller design explicitly uses dropout probabilities.
} 
domain representation motivates the formulation of a noiseshaping model of the NCS, which we use for rate-distortion analysis.

The key novelty of the present work (when compared, e.g., with [20]) is that it examines a NCS with a communication channel which is both bit-rate limited and unreliable, and where the plant is affected by random disturbances with possibly unbounded support. Furthermore, our approach goes beyond studying stability, and gives insight into closed loop performance in the presence of random packet dropouts and disturbances for a given expected bit-rate. Our results show that the use of larger prediction horizons will, in general, lead to enhanced stability and performance properties.

The remainder of this paper is organized as follows: In Sections II and III, we present the NCS of interest. Section IV then establishes results on stochastic stability. In Section V, we study closed loop performance of the NCS. Here, we characterize spectral densities and also assess the bit-rate required to guarantee a desired performance level. Section VI draws conclusions. Proofs of the main technical results are included in appendices.

\section{Notation}

$I$ denotes the identity matrix of appropriate dimension; the superscripts ${ }^{T}$ and ${ }^{H}$ refer to transpose and Hermitian transposition, respectively. For any square matrix $K$, $\operatorname{det} K$ denotes its determinant, $\operatorname{tr} K$ its trace, eigs $K$ its eigenvalues; its spectral norm $\|K\|$ is given by $\|K\|^{2}=\max \operatorname{eigs}\left(K^{H} K\right)$; $\rho(K)=\max |\operatorname{eigs} K|$ denotes its spectral radius, $\mu(K)$ its largest positive eigenvalue. We say that $K$ is (Schur-) stable, if and only if $\rho(K)<1 . K \succeq 0$ means that $K$ is positive semi-definite, $\succ$ refers to positive-definiteness. For any vector $x$ and any square matrix $K$, we define $\|x\|_{K}^{2}=x^{T} K x$ and $\|x\|^{2}=x^{T} x$. The symbol $\otimes$ denotes the Kronecker product. The natural numbers are denoted via $\mathbb{N}$, whereas $\mathbb{N}_{0} \triangleq\{0,1,2, \ldots\}$. We adopt the conventions $0^{0}=1$ and $\sum_{\ell=0}^{-1} a_{\ell}=0$, for all $a_{\ell}$.

For any asymptotically stationary real stochastic processes $\zeta=\left\{\zeta_{k}\right\}$ and $\eta=\left\{\eta_{k}\right\}$, we define the cross-covariance function $R_{\zeta \eta}(\ell) \triangleq \lim _{k \rightarrow \infty} \mathbb{E}\left\{\zeta_{k+\ell} \eta_{k}^{T}\right\}$ and the covariance function $R_{\zeta}(\ell) \triangleq \lim _{k \rightarrow \infty} \mathbb{E}\left\{\zeta_{k+\ell} \zeta_{k}^{T}\right\}$, where $\mathbb{E}(\cdot)$ is the expectation operator. We furthermore let $\mathbb{E}_{x}(\cdot)$ denote conditional expectation given $x$. Differential entropy is denoted by $h(\cdot)$, conditional discrete entropy by $H(\cdot \mid \cdot)$, mutual information by $I(\cdot, \cdot)$ and Divergence by $\mathcal{D}(\cdot \| \cdot)$; see, e.g., [27]. (Cross-) spectra are defined as the two-sided $z$-Transform of the (cross-) covariance functions. When evaluating spectra on the unit circle, we will use the term spectral densities, see, e.g., [28]. Finally, if $\zeta$ is zero-mean Gaussian distributed with covariance matrix $R_{\zeta}(0)$, then we write $\zeta \sim \mathcal{N}\left(0, R_{\zeta}(0)\right)$.

\section{Packetized Control over Erasure Channels}

We consider the following discrete-time stochastic linear time invariant (LTI) possibly unstable dynamical plant model with state $x_{k} \in \mathbb{R}^{n}, n \geq 1$ and scalar input $u_{k} \in \mathbb{R}$ :

$$
x_{k+1}=A x_{k}+B_{1} u_{k}+B_{2} w_{k} .
$$

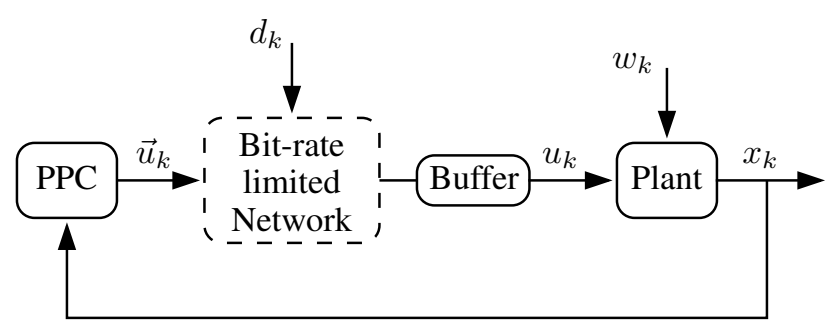

Fig. 1. NCS architecture with buffer at the actuator node

In (1), $w_{k} \in \mathbb{R}^{m}, m \geq 1$, is an unmeasured disturbance, modelled as an arbitrarily distributed (and with possibly unbounded support) zero-mean stochastic process with spectral density $S_{w}\left(e^{j \omega}\right)$ and covariance matrix $R_{w}(0)$. The initial state $x_{0}$ is arbitrarily distributed, having covariance matrix $R_{x}(0)$. We assume that the pair $\left(A, B_{1}\right)$ is stabilizable.

\section{A. Network Effects}

Our interest lies in clock-driven Ethernet-like networks situated between controller output and plant input. Thus, all data to be transmitted needs to be quantized and is sent in large time-stamped packets. Due to transmission errors and congestion, the network introduces packet dropouts and timedelays. We model the network as an erasure channel, which operates at the same sampling-rate as the plant model $^{3}$. In the sequel, we describe transmission effects via the discrete Bernoulli process $\left\{d_{k}\right\}$ defined via:

$d_{k} \triangleq \begin{cases}1, & \text { if packet dropout occurs at instant } k, \\ 0, & \text { if packet dropout does not occur at instant } k .\end{cases}$

We will assume that each variable $d_{k}$ is i.i.d. with distribution

$$
\operatorname{Prob}\left(d_{k}=1\right)=p, \quad \operatorname{Prob}\left(d_{k}=0\right)=1-p,
$$

where $p \in(0,1)$ is the dropout-rate. In practical situations, $p$ is not known exactly. Accordingly, we will study a design problem, where the controller does not have knowledge about $p$. (Of course, closed loop stability and performance will depend upon $p$, see Sections IV and V.)

As foreshadowed in the introduction, at each time instant $k$, the PPC sends a control packet, say $\vec{u}_{k}$, to the plant input node. To achieve good performance, despite unreliable communication, $\vec{u}_{k}$ contains possible control inputs for a finite number of $N$ future time instants. At the plant input side, the received packets are buffered, providing the plant inputs, see Fig. 1. We will next describe the buffering procedure. In Section III we will then show how to design $\vec{u}_{k}$.

\section{B. Buffering}

The buffering mechanism amounts to a parallel-in serialout shift register, which acts as a safeguard against packet dropouts. For that purpose, the buffer state, say $b_{k} \in \mathbb{R}^{N}$, is overwritten whenever a valid (i.e., uncorrupted and undelayed) control packet arrives. Actuator values are passed on to the

\footnotetext{
${ }^{3}$ Small fixed time-delays can be included in (1). Signals, which are delayed more, are then considered as "lost."
} 
plant sequentially until the next valid control packet $\vec{u}_{k}$ is received, see Fig. 1. More formally, we have:

$$
\begin{aligned}
b_{k} & =d_{k} M b_{k-1}+\left(1-d_{k}\right) \vec{u}_{k}, \\
u_{k} & =e_{1}^{T} b_{k},
\end{aligned}
$$

where

$$
M \triangleq\left[\begin{array}{ccccc}
0 & 1 & 0 & \ldots & 0 \\
\vdots & \ddots & \ddots & \ddots & \vdots \\
0 & \ldots & 0 & 1 & 0 \\
0 & \ldots & \ldots & 0 & 1 \\
0 & \ldots & \ldots & \ldots & 0
\end{array}\right] \in \mathbb{R}^{N \times N}, e_{1}=\left[\begin{array}{c}
1 \\
0 \\
\vdots \\
\vdots \\
0
\end{array}\right] \in \mathbb{R}^{N \times 1},
$$

see, e.g., [20].

Remark 1: The choice of $M$ in (4) corresponds to setting the buffer state to zero if no data is received over $N$ consecutive instants. Alternatively, if one wished to hold the latest value, one could set the "last" element of $M$ equal to 1 . In [22] these choices are compared for buffers with $N=1$.

For further reference, we define the augmented state $\theta_{k}$ via:

$$
\theta_{k} \triangleq\left[\begin{array}{c}
x_{k} \\
b_{k-1}
\end{array}\right] \in \mathbb{R}^{n+N} .
$$

Equations (1) and (3) then allow us to describe the NCS via:

$\theta_{k+1}=\left[\begin{array}{cc}A & d_{k} B_{1} e_{1}^{T} M \\ 0 & d_{k} M\end{array}\right] \theta_{k}+\left[\begin{array}{c}B_{1} e_{1}^{T} \\ I\end{array}\right]\left(1-d_{k}\right) \vec{u}_{k}+\left[\begin{array}{c}B_{2} \\ 0\end{array}\right] w_{k}$.

Thus, if $\vec{u}_{k}$ depends linearly upon the state vector $\theta_{k}$, then the NCS architecture studied here fits into the class of Markov jump-linear systems (MJLS's); see, e.g., [25], [26], [29], and also [30]-[33] for other NCS architectures which can be characterized as MJLS's.

A key difficulty of the situation at hand is that, since there are no acknowledgments of receipt, the controller does not have certainty about whether previous packets have arrived at the plant side. Therefore, the jump variable $d_{k}$ and the buffer contents $b_{k-1}$ are unavailable for the calculation of $\vec{u}_{k}$.

In the following section, we will present a control packet design method, which uses elements of quantized predictive control and is suitable for bit-rate limited networks without acknowledgments and where packet dropout probabilities are unknown.

Remark 2: Whilst the absence of acknowledgments implies that controllers studied, e.g., in [16], [19], [25], [30], [34][37], cannot be employed directly, some progress on predictive control of MJLS's without observation of the jump variable has been reported, e.g., in [38]-[40]. How to adapt these methods to the present situation of quantized packetized predictive control remains the subject of further study.

\section{QUANTIZED PPC}

We now turn our attention to controller design. Here, we will assume that the controller has access to the plant state (but not to the buffer state). Furthermore, the controller does not know the dropout-rate $p$. Within this challenging scenario, control packets $\vec{u}_{k}$ are formed by adapting a quantized predictive control approach as studied in [41] and described next.

\section{A. Quantized Predictive Control}

In the quantized predictive control formulation of [41], at each time instant $k$ and for a given plant state $x_{k}$, the following cost function is minimized:

$$
J\left(\vec{u}^{\prime}, x_{k}\right) \triangleq\left\|x_{N}^{\prime}\right\|_{P}^{2}+\sum_{\ell=0}^{N-1}\left(\left\|x_{\ell}^{\prime}\right\|_{Q}^{2}+\lambda\left(u_{\ell}^{\prime}\right)^{2}\right) .
$$

In (7), $x_{\ell}^{\prime}$ are predicted plant states for inputs $u_{\ell}^{\prime}$. The horizon length $N \geq 1$ is taken equal to the buffer size, whereas the design variables $P \succeq 0, Q \succeq 0$ and $\lambda>0$ allow one to tradeoff control performance versus control effort. These design parameters influence closed loop stability and performance, see also [42]. In Section IV we will investigate how to design these variables for the networked situation of interest.

The cost function in (7) examines a prediction of the plant model over a finite horizon of length $N$. The predicted state trajectories at time $k$ are generated by

$$
x_{\ell+1}^{\prime}=A x_{\ell}^{\prime}+B_{1} u_{\ell}^{\prime}, \quad \ell \in\{0,1, \ldots, N-1\}
$$

with $x_{0}^{\prime}=x_{k}$, the current plant state. The entries in

$$
\vec{u}^{\prime}=\left[\begin{array}{lll}
u_{0}^{\prime} & \ldots & u_{N-1}^{\prime}
\end{array}\right]^{T}
$$

represent the associated predicted plant inputs.

An important aspect of the NCS studied is that the network is bit-rate limited. This introduces a quantization constraint into the problem of minimizing $J\left(\vec{u}^{\prime}, x_{k}\right)$. A closed form solution to this problem was derived in [41]. To state the result, we introduce $\bar{Q} \triangleq \operatorname{diag}(Q, \ldots, Q, P)$ and:

$$
\Phi \triangleq\left[\begin{array}{cccc}
B_{1} & 0 & \ldots & 0 \\
A B_{1} & B_{1} & \ldots & 0 \\
\vdots & \vdots & \ddots & \vdots \\
A^{N-1} B_{1} & A^{N-2} B_{1} & \ldots & B_{1}
\end{array}\right], \Upsilon \triangleq\left[\begin{array}{c}
A \\
A^{2} \\
\vdots \\
A^{N}
\end{array}\right] .
$$

Theorem 1 ([41]): Consider any finite or countably infinite quantized set $\mathbb{U} \subset \mathbb{R}^{N}$, and define:

$$
\xi_{k} \triangleq \Gamma x_{k}, \quad \Gamma \triangleq-\Psi^{-T} \Phi^{T} \bar{Q} \Upsilon,
$$

where $\Psi \in \mathbb{R}^{N \times N}$ is obtained from the factorization

$$
\Psi^{T} \Psi=\Phi^{T} \bar{Q} \Phi+\lambda I .
$$

Then the constrained optimizer $\vec{u}_{k}=\arg \min _{\vec{u}^{\prime} \in \mathbb{U}} J\left(\vec{u}^{\prime}, x_{k}\right)$, see (7), satisfies:

$$
\vec{u}_{k}=\Psi^{-1} q\left(\xi_{k}\right),
$$

where $q(\cdot)$ is the nearest neighbour (Euclidean) vector quantizer with alphabet $\Psi \mathbb{U}$.

Proof: The proof mirrors that of Theorem 1 in [41].

In [41] the quantizer alphabet was assumed given, whereas in the present work it can be designed. In fact, in NCS's quantization constraints arise due to the need to accommodate bitrate limitations of the network. Thus, quantization alphabets constitute an important design parameter. 


\section{B. Control Packet Design}

Theorem 1 shows that one can work in a domain where nearest neighbour vector quantization of $\xi_{k}$ is optimal. Therefore, in addition to choosing the parameters of the cost function in (7), designing a quantized PPC for the system (1) encompasses choosing a vector quantizer (VQ) for $\xi_{k}$, i.e., designing the alphabet $\Psi \mathbb{U}$; see, e.g., [43].

Deterministic VQs, as given in (10), are generally not amenable for analysis of the trade-off between expected bitrates and distortions. On the other hand, some stochastic quantization strategies allow for analysis and design which takes into account rate-distortion issues. In particular, in the sequel we will focus our attention on (entropy-coded) dithered lattice VQs, hereafter abbreviated ECDQs. This amounts to replacing the solution in (10) by:

$$
\vec{u}_{k}=\Psi^{-1}\left(q_{\mathcal{L}}\left(\xi_{k}+\eta_{k}\right)-\eta_{k}\right),
$$

where $q_{\mathcal{L}}$ is a nearest neighbour lattice VQ with lattice $\mathcal{L}{ }^{4}$ The dither $\eta_{k}$ in (11) is taken as a (pseudo-) random zeromean i.i.d. vector process. The current dither vector is chosen independent of current and past values of the input signal and is uniformly distributed over the basic Voronoi cell of the lattice VQ. The dither process is known to the encoder as well as the decoder; see, e.g., [47], [48]. Hence, transmitting $\vec{u}_{k}$ is equivalent to transmitting $q_{\mathcal{L}}\left(\xi_{k}+\eta_{k}\right)$, which, in effect, amounts to sending a codeword-index associated to an element of $\Psi \mathbb{U}$. Since the ECDQ alphabet is given by the points of the lattice $\mathcal{L}$, the resulting alphabet (in the $\vec{u}$-domain) is given by the shaped lattice $\mathbb{U}=\Psi^{-1} \mathcal{L}$.

A key property is that ECDQs satisfy:

Assumption 1: The following linear additive noise model holds:

$$
q_{\mathcal{L}}\left(\xi_{k}+\eta_{k}\right)-\eta_{k}=\xi_{k}+n_{k},
$$

where $n_{k}$ is distributed as $-\eta_{k}$, which gives that $\mathbb{E}\left\{\left\|n_{k}\right\|^{2}\right\}=$ $\mathbb{E}\left\{\left\|\eta_{k}\right\|^{2}\right\}$. In addition, each $n_{k}$ is a zero-mean white vector with variance

$$
\sigma_{n}^{2}=\operatorname{tr} R_{n}(0)
$$

and is independent of $\xi_{k-\ell}, \forall \ell \geq 0$.

Given Assumption 1, for a given dimension $N$ and lattice $\mathcal{L}$, designing an ECDQ reduces to finding an appropriate value of the quantization noise variance $\sigma_{n}^{2}$. In fact, we have:

$$
\sigma_{n}^{2}=N \mathcal{G V}^{2 / N}
$$

where $\mathcal{V}$ is the volume of the Voronoi cell and $\mathcal{G}$ is its dimensionless normalized second moment of inertia. Thus, the noise variance $\sigma_{n}^{2}$ determines $\mathcal{V}$ and thereby completely specifies the VQ. In general, to achieve lower values of $\sigma_{n}^{2}$, larger bitrates are required. In Section V-B we will further elucidate

\footnotetext{
${ }^{4}$ For any finite dimension $N$ and (expected) bit-rate, it is known that, optimal entropy-constrained VQs minimize the expected distortion. These VQs can be designed, e.g., via the LBG algorithm [43]. Unfortunately, the resultant quantizers usually lack structure and, thus, search complexity is substantial. Furthermore, redesign is required for different bit-rates. On the contrary, lattice VQs have fast implementations and often give near optimal performance; see, e.g., [44]. This makes ECDQs a practical alternative for closed loop control, where computations should be kept bounded, see also discussions in [45]. Rate-distortion optimal lattice VQs in various dimensions (including fast encoding strategies) are specified in [46].
}

the tradeoff between average bit-rates of the quantized signal transmitted and the achieved closed loop performance.

Following the ideas underlying PPCs, see, e.g., [17], at each time instant $k$, and for current state $x_{k}$, the controller sends the entire sequence $\vec{u}_{k}$ to the actuator node. As explained in Section II-B, depending upon future packet dropout scenarios, a subsequence of $\vec{u}_{k}$ will be applied at the plant input, or not. As in the receding horizon paradigm, at the next time instant, $x_{k+1}$ is used to calculate $\vec{u}_{k+1}$, etc.

Remark 3: It is well-known that it is often useful to include pre- and post-filtering around the quantizer. How to design optimal pre- and post-filters for closed loop applications has been treated partially in [49]-[51]. We note that any causal LTI filtering can be easily incorporated into our framework by augmenting the plant model (1) with the post-filter. The pre-filter is implicit in the controller.

\section{Stochastic Stability of Quantized Packetized PREDICTIVE CONTROL}

To investigate closed loop stability of the NCS, we will exploit the fact that the system under study constitutes a MJLS. In fact, given (5), (6), (11) and (12), the NCS with quantization, dropouts and disturbances can be described via:

$$
\theta_{k+1}=\bar{A}\left(d_{k}\right) \theta_{k}+\bar{B}\left(d_{k}\right) \nu_{k}
$$

where $\nu_{k}^{T}=\left[\begin{array}{ll}w_{k}^{T} & n_{k}^{T}\end{array}\right]$ and where

$$
\begin{gathered}
\bar{A}(0)=\left[\begin{array}{cc}
A+B_{1} e_{1}^{T} \Psi^{-1} \Gamma & 0 \\
\Psi^{-1} \Gamma & 0
\end{array}\right], \bar{A}(1)=\left[\begin{array}{cc}
A & B_{1} e_{1}^{T} M \\
0 & M
\end{array}\right], \\
\bar{B}(0)=\left[\begin{array}{cc}
B_{2} & B_{1} e_{1}^{T} \Psi^{-1} \\
0 & \Psi^{-1}
\end{array}\right], \bar{B}(1)=\left[\begin{array}{cc}
B_{2} & 0 \\
0 & 0
\end{array}\right] .
\end{gathered}
$$

\section{A. Basic Definitions and Results}

Several works have studied stability of MJLS's; see, e.g., [25], [26], [29], [52]. In particular, from [25], [52], we will adopt the following notions of stationarity and stability: ${ }^{5}$

Definition 1 (AWSS): System (15) is asymptotically widesense stationary (AWSS), if for all initial conditions $d_{0} \in$ $\{0,1\}$ and $\theta_{0}$ with bounded variance, there exist bounded $\mu$ and $R_{\theta}(\ell)$ independent of $\left(\theta_{0}, d_{0}\right)$ such that

$$
\begin{aligned}
\lim _{k \rightarrow \infty} \mathbb{E}\left\{\theta_{k}\right\} & =\mu \\
\lim _{k \rightarrow \infty} \mathbb{E}\left\{\theta_{k+\ell} \theta_{k}^{T}\right\} & =R_{\theta}(\ell), \quad \forall \ell \geq 0 .
\end{aligned}
$$

Definition 2 (MSS): System (15) is mean-square stable, if for all $d_{0} \in\{0,1\}$ and $\theta_{0}$ with bounded variance, there exist bounded $\mu$ and $R_{\theta}(0)$ independent of $\left(\theta_{0}, d_{0}\right)$ such that

$$
\begin{aligned}
\lim _{k \rightarrow \infty} \mathbb{E}\left\{\theta_{k}\right\} & =\mu \\
\lim _{k \rightarrow \infty} \mathbb{E}\left\{\theta_{k} \theta_{k}^{T}\right\} & =R_{\theta}(0) .
\end{aligned}
$$

Theorem 2 included below gives necessary and sufficient conditions for AWSS and MSS of MJLS's. It encompasses various results documented in the literature.

\footnotetext{
${ }^{5}$ It was shown in [26] that for the homogeneous MJLS (17), MSS is equivalent to stochastic stability and exponential MSS, and that MSS is also sufficient for almost sure stability. Clearly, AWSS implies MSS.
} 
Theorem 2 (Adapted from [25]): Consider (15) and its homogeneous counterpart:

$$
\tilde{\theta}_{k+1}=\bar{A}\left(d_{k}\right) \tilde{\theta}_{k}, \quad \tilde{\theta}_{k} \triangleq\left[\begin{array}{c}
\tilde{x}_{k} \\
\tilde{b}_{k-1}
\end{array}\right],
$$

see (5). Then the following assertions are equivalent:

1) System (15) is MSS.

2) System (15) is AWSS.

3) System (17) is MSS.

4) For all initial conditions $d_{0} \in\{0,1\}$ and $\tilde{\theta}_{0}$ with bounded variance, it holds that

$$
\sum_{k=0}^{\infty} \mathbb{E}\left\{\left\|\tilde{\theta}_{k}\right\|^{2}\right\}<\infty
$$

5) There exists $\Lambda(p) \succ 0$ which satisfies the Linear Matrix Inequality:

$$
\Lambda(p)-(1-p) \bar{A}(0) \Lambda(p) \bar{A}^{T}(0)-p \bar{A}(1) \Lambda(p) \bar{A}^{T}(1) \succ 0 .
$$

6) The following matrix is Schur:

$$
(1-p) \bar{A}(0) \otimes \bar{A}(0)+p \bar{A}(1) \otimes \bar{A}(1) .
$$

Proof: In the i.i.d. dropout case (2), (15) constitutes an MJLS with transition probability matrix $\left[\begin{array}{ll}1-p & p \\ 1-p & p\end{array}\right]$. The result now follows from Theorems 3.9, 3.26 and 3.33 in [25].

It is worth noting that, provided Assumption 1 holds, stationarity and stability of the NCS is independent of external disturbances and quantization noise with bounded variance. Whilst the latter is linked to the bit-rate of the quantizer, MSS and AWSS cannot be guaranteed for arbitrary bit-rates, see also [2]. This issue will be further discussed in Section V-B.

Lemma 1 stated below gives a necessary condition for MSS (see Definition 2) of MJLS's of the form (15). It gives a bound on the packet dropout-rate which only depends on the plant model (1) and not on how the control packet $\vec{u}_{k}$ is designed. ${ }^{6}$

Lemma 1: If the system (15) is MSS, then the dropout-rate is bounded by the spectral radius of the plant model $A$ via:

$$
p<\frac{1}{\rho(A)^{2}} .
$$

Proof: By [54, Theorem 2.1] (see also [29, Remark 2]), a necessary condition for MSS of (17) is that $\sqrt{p} \bar{A}(1)$ be stable. Equation (16) gives that $\operatorname{det}(z I-\sqrt{p} A(1))=\operatorname{det}(z I-$ $\sqrt{p} M) \operatorname{det}(z I-\sqrt{p} A)=z^{N} \operatorname{det}(z I-\sqrt{p} A)$. The result now follows from using Theorem 2.

In the sequel we will build upon the above results to study MSS and AWSS of the packetized predictive NCS with dropouts, disturbances and quantization. For that purpose, we will first investigate expressions (19) and (20). Then, in Section IV-C, we will present an analysis method which exploits the fact that the controller minimizes the cost function (7).

\footnotetext{
${ }^{6}$ As shown in [22] (and compare to [23], [53]), in some situations, the bound in (21) can be achieved by a stochastic control formulation, which uses knowledge of the dropout-rate $p$. In contrast, in the present work we are interested in situations where the controller does not have knowledge of $p$.
}

\section{B. Stability Analysis via Expressions (19) and (20)}

If we assume that the cost function (7) is chosen such that the closed loop system without dropouts is MSS, then stability analysis can be cast as identifying dropout-rates which preserve stability. In terms of the model (16) and in view of Theorem 2, requiring MSS when there are no dropouts amounts to making the following assumption:

Assumption 2: The controller is such that $\bar{A}(0)$ is Schur. $\square$

Remark 4: It is worth noting that Assumption 2 will hold, for example, if $P$ in (7) is chosen as the positive definite solution to the algebraic Riccati equation

$$
P=A^{T} P A+Q-K^{T}\left(\lambda I+B_{1}^{T} P B_{1}\right) K,
$$

where $K=-\left(\lambda I+B_{1}^{T} P B_{1}\right)^{-1} B_{1}^{T} P A$; see, e.g., [42].

Perhaps not surprisingly, the buffered NCS architecture under study preserves stability if dropout-rates are small, as stated in the following lemma:

Lemma 2: Suppose that Assumptions 1 and 2 hold. Then there exists a dropout-rate $p^{\star}>0$ such that the NCS (15) is MSS and AWSS for all dropout-rates $p \in\left(0, p^{\star}\right)$.

Proof: By Assumption 2, for any $S \succ 0$ there exists $\Omega \succ 0$, such that $\Omega-\bar{A}(0) \Omega \bar{A}^{T}(0)=S$. In particular, we can set $S=I$ and replace the associated solution $\Omega$ in (19) to obtain the following sufficient condition for MSS and AWSS:

$$
\begin{aligned}
& \Omega-(1-p) \bar{A}(0) \Omega \bar{A}^{T}(0)-p \bar{A}(1) \Omega \bar{A}^{T}(1) \\
& \quad=I-p\left(\bar{A}(1) \Omega \bar{A}^{T}(1)-\bar{A}(0) \Omega \bar{A}^{T}(0)\right) \succ 0 .
\end{aligned}
$$

By [55, Lemma 8.4.1], the condition (23) is satisfied if and only if $0<p \leq p^{\star}$, where $1 / p^{\star}>\max \operatorname{eigs}\left(\bar{A}(1) \Omega \bar{A}^{T}(1)-\right.$ $\left.\bar{A}(0) \Omega \bar{A}^{T}(0)\right)$. With this choice, $\forall p \in\left(0, p^{\star}\right)$ there exists a solution to (19). The result now follows from Theorem 2.

Given Lemma 2, the question arises of finding the largest dropout-rate such that the NCS is MSS and AWSS. The MJLS description (17) allows us to use results in [32] to obtain the following characterization of the maximum dropout-rate which preserves MSS and AWSS:

Lemma 3: Suppose that Assumption 2 holds and define

$$
\mathcal{W} \triangleq\left[\begin{array}{cc}
(\mathcal{U} \otimes \tilde{\mathcal{U}}+\tilde{\mathcal{U}} \otimes \mathcal{U})(I-\mathcal{U} \otimes \mathcal{U})^{-1} & \tilde{\mathcal{U}} \otimes \tilde{\mathcal{U}} \\
(I-\mathcal{U} \otimes \mathcal{U})^{-1} & 0
\end{array}\right]
$$

where

$$
\mathcal{U} \triangleq \bar{A}(0) \otimes \bar{A}(0), \quad \tilde{\mathcal{U}} \triangleq \bar{A}(1) \otimes \bar{A}(1)-\bar{A}(0) \otimes \bar{A}(0) .
$$

Then the NCS (15) is MSS and AWSS $\forall p \in\left[0, p^{\star}\right)$, iff

$$
p^{\star} \leq 1 / \mu(\mathcal{W}) .
$$

Proof: Follows from [32, Lemma 2 and Theorem 6].

The above lemma gives maximum dropout-rates which preserve stability in terms of Kronecker products. Unfortunately, for large horizon lengths $N$ or plant orders $n$, evaluating (24) becomes computationally infeasible, due to the need to invert and find eigenvalues of matrices of large dimensions, which often are ill-conditioned. (The matrix $\mathcal{U} \otimes \mathcal{U}$ has $(N+n)^{4}$ rows and columns.) The analysis included in the sequel gives stability characterizations, which involve only matrices of moderate dimensions. 
We begin by introducing the expected system matrices:

$$
\begin{aligned}
& \mathcal{A}(p) \triangleq \mathbb{E}\left\{\bar{A}\left(d_{k}\right)\right\}=(1-p) \bar{A}(0)+p \bar{A}(1) \\
& \mathcal{B}(p) \triangleq \mathbb{E}\left\{\bar{B}\left(d_{k}\right)\right\}=(1-p) \bar{B}(0)+p \bar{B}(1),
\end{aligned}
$$

where we have used (15) and conditioning on $d_{k}$. Equation (16) gives that

$$
\mathcal{A}(p)=\left[\begin{array}{cc}
A+(1-p) B_{1} e_{1}^{T} \Psi^{-1} \Gamma & p B_{1} e_{1}^{T} M \\
(1-p) \Psi^{-1} \Gamma & p M
\end{array}\right],
$$

whereas $\mathcal{B}(p)=\left[\begin{array}{ll}\mathcal{B}_{w} & \mathcal{B}_{n}(p)\end{array}\right]$ with

$$
\mathcal{B}_{w} \triangleq\left[\begin{array}{c}
B_{2} \\
0
\end{array}\right], \mathcal{B}_{n}(p) \triangleq\left[\begin{array}{c}
(1-p) B_{1} e_{1}^{T} \Psi^{-1} \\
(1-p) \Psi^{-1}
\end{array}\right] .
$$

The following properties of $\mathcal{A}(p)$ are easy to prove:

Lemma 4: If (15) is MSS, then $p$ is such that $\mathcal{A}(p)$ is Schur.

Proof: By conditioning on $d_{k}$, we can use (15) to form the recursion

$\mathbb{E}\left\{\theta_{k+1}\right\}=(1-p) \bar{A}(0) \mathbb{E}\left\{\theta_{k}\right\}+p \bar{A}(1) \mathbb{E}\left\{\theta_{k}\right\}=\mathcal{A}(p) \mathbb{E}\left\{\theta_{k}\right\}$,

which converges, if and only if $\rho(\mathcal{A}(p))<1$.

Lemma 5: Suppose that Assumption 2 holds. Then there exists a dropout-rate $p^{\sharp}>0$ such that $\mathcal{A}(p)$ is Schur for all $p \in\left(0, p^{\sharp}\right)$.

Proof: Uses a similar perturbation argument to that used in the proof of Lemma 1.

Motivated by Statement 5) of Theorem 2 and the approach used in [24] and [56] for NCS's with scalar transmission, we can derive sufficient conditions for stability of the NCS as stated in Lemma 7. To state this result, we first require a more general characterization which gives sufficient (but not necessary) conditions of MSS and AWSS of the NCS.

Lemma 6: Suppose that the dropout-rate $p$ is such that $\mathcal{A}(p)$ is Schur and define:

$$
\widetilde{\mathcal{A}} \triangleq \bar{A}(1)-\bar{A}(0)=\left[\begin{array}{cc}
-B_{1} e_{1}^{T} \Psi^{-1} \Gamma & B_{1} e_{1}^{T} M \\
-\Psi^{-1} \Gamma & M
\end{array}\right] .
$$

If there exist $\Theta \succeq 0$ and $\Xi(p) \succeq 0$ which satisfy: ${ }^{7}$

$$
\begin{gathered}
p(1-p) \widetilde{\mathcal{A}} \Xi(p) \widetilde{\mathcal{A}}^{T} \prec \Theta, \\
\mathcal{A}(p) \Xi(p) \mathcal{A}(p)^{T}-\Xi(p)+\Theta=0,
\end{gathered}
$$

then the MJLS (15) is MSS and AWSS.

Proof: See Appendix A.

Lemma 7: If $p$ is such that $\mathcal{A}(p)$ is Schur-stable and

$$
p^{-1}>1+\left\|\left[\begin{array}{ll}
\Gamma & -\Psi M
\end{array}\right] \Xi_{n}(p)\left[\begin{array}{ll}
\Gamma & -\Psi M
\end{array}\right]^{T}\right\|,
$$

where $\Xi_{n}(p)$ solves the Lyapunov equation

$$
\mathcal{A}(p) \Xi_{n}(p) \mathcal{A}(p)^{T}-\Xi_{n}(p)+\mathcal{B}_{n}(p) \mathcal{B}_{n}(p)^{T}=0,
$$

then the MJLS (15) is MSS and AWSS.

Proof: $\quad$ By (26)-(28), we can write $\widetilde{\mathcal{A}}=\widetilde{\mathcal{A}_{1}} \widetilde{\mathcal{A}}_{2}$ with $\widetilde{\mathcal{A}}_{1}=\mathcal{B}_{n}(p)$ whereas $\widetilde{\mathcal{A}}_{2}=(1-p)^{-1}\left[\begin{array}{ll}-\Gamma & \Psi M\end{array}\right]$. We next use Lemma 6 and set $\Theta=\widetilde{\mathcal{A}}_{1} \widetilde{\mathcal{A}}_{1}^{T} \succeq 0$. If we now write $\Xi(p)=\Xi_{n}(p)$, then (30) becomes (32) and (29) amounts to

$$
0 \prec \widetilde{\mathcal{A}}_{1}\left(I-p(1-p) \widetilde{\mathcal{A}}_{2} \Xi_{n}(p) \widetilde{\mathcal{A}}_{2}^{T}\right) \widetilde{\mathcal{A}}_{1}^{T},
$$

\footnotetext{
${ }^{7}$ Since $\mathcal{A}(p)$ is assumed Schur-stable, (30) has a unique positive definite solution, namely $\Xi(p)=\sum_{i=0}^{\infty} \mathcal{A}(p)^{i} \Theta\left(\mathcal{A}(p)^{T}\right)^{i}$.
}

which holds if $I-p(1-p) \widetilde{\mathcal{A}}_{2} \Xi_{n}(p) \widetilde{\mathcal{A}}_{2}^{T} \succ 0$. By [55, Lemma 8.4.1], the latter condition holds if and only if

$$
p(1-p) \max \operatorname{eigs}\left(\widetilde{\mathcal{A}}_{2} \Xi_{n}(p) \widetilde{\mathcal{A}}_{2}^{T}\right)<1,
$$

which is equivalent to (31).

Lemma 7 gives insight into the stability issue of packetized NCS with quantization, disturbances and Bernoulli dropouts. Interestingly, as we will see in Section V-A, the matrix $\Xi_{n}(p)$ introduced in (32) also plays a role in quantifying the impact of the quantization noise $\left\{n_{k}\right\}$ on NCS performance.

\section{Stochastic Stability via the Optimal Value Function}

The stability characterizations of Section IV-B are applicable to any packetized NCS which can be described by (15) and (16), where $\Gamma, \Psi$ and $M$ are arbitrary matrices of appropriate dimensions. We will next derive more specific results. These exploit the fact that the packetized predictive control algorithm minimizes the cost function (7).

By Theorem 2, we only need to examine the NCS without quantization effects and disturbances. Motivated by our analysis in [20], we will denote the time instants where there are no packet dropouts, i.e., where $d_{k}=0$, as

$$
\mathcal{T}=\left\{k_{i}\right\}_{i \in \mathbb{N}_{0}} \subseteq \mathbb{N}_{0}, \quad k_{i+1}>k_{i}, \quad \forall i \in \mathbb{N}_{0}
$$

and examine the sequence of optimal costs of the unconstrained optimization problem at the instants of successful transmission, namely

$$
V\left(\tilde{x}_{k_{i}}\right) \triangleq \min _{\vec{u}^{\prime} \in \mathbb{R}^{N}} J\left(\vec{u}^{\prime}, \tilde{x}_{k_{i}}\right), \quad k_{i} \in \mathcal{T},
$$

see (7) and (17). The quadratic program (34) has been extensively studied; see, e.g., [57]. In particular, it holds that:

$$
V\left(\tilde{x}_{k_{i}}\right)=\left\|\tilde{x}_{k_{i}}\right\|_{P_{0}}^{2},
$$

where $P_{0}$ is obtained by iterating the discrete-time Riccati Equation:

$P_{\ell}=A\left(P_{\ell+1}-P_{\ell+1} B_{1}\left(B_{1}^{T} P_{\ell+1} B_{1}+\lambda I\right)^{-1} B_{1}^{T} P_{\ell+1}\right) A+Q$,

where $\ell \in\{0,1, \ldots, N-1\}$ and starting from $P_{N}=P$.

Lemma 8 stated below gives a closed form expression for the expected value of the optimal cost function at time $k_{1}$ for given state $\tilde{x}_{k_{0}}$. To state our result, we introduce the mappings $\phi_{\ell}: \mathbb{R}^{n} \mapsto \mathbb{R}^{n}$, where $\ell \in\{1,2, \ldots, N\}$ and which are defined recursively via

$$
\begin{aligned}
\phi_{\ell+1} & =\left(A+B_{1} K_{\ell}\right) \phi_{\ell}, \quad \ell \in\{1,2, \ldots, N-1\} \\
\phi_{1} & =A+B_{1} K_{0}
\end{aligned}
$$

where

$K_{\ell} \triangleq-\left(B_{1}^{T} P_{\ell+1} B_{1}+\lambda I\right)^{-1} B_{1} P_{\ell+1} A, \ell \in\{0,1, \ldots, N-1\}$.

We will assume that $p$ satisfies (21), denote via $\Omega(p) \succeq 0$ the solution of the Lyapunov equation

$$
\Omega(p)=p A^{T} \Omega(p) A+P_{0}
$$


and also define:

$$
\Pi(p, N) \triangleq(1-p)\left(p^{N-1} \phi_{N}^{T} \Omega(p) \phi_{N}+\sum_{i=1}^{N-1} p^{i-1} \phi_{i}^{T} P_{0} \phi_{i}\right) .
$$

Lemma 8: Consider $k_{0}, k_{1} \in \mathcal{T}$ and suppose that $p$ satisfies (21). We then have

$$
\mathbb{E}_{\tilde{x}_{k_{0}}} V\left(\tilde{x}_{k_{1}}\right)=\tilde{x}_{k_{0}}^{T} \Pi(p, N) \tilde{x}_{k_{0}}
$$

Proof: See Appendix B.

Despite the fact that the state of the NCS is $\theta_{k}$ and not $\tilde{x}_{k}$, see (5), Lemma 8 can be used for the design of the cost function parameters in (7) such that the NCS (with dropouts and non-zero $w_{k}$ and $n_{k}$ ) is MSS and AWSS. Our main stability result is stated as follows:

Theorem 3: Suppose that Assumption 1 holds and that the dropout-rate $p$ satisfies (21). If

$$
P_{0}-\Pi(p, N) \succeq 0,
$$

then the NCS in (15)-(16) is MSS and AWSS.

Proof: See Appendix C.

As a consequence of this theorem, MSS of the NCS is closely linked to the matrix $\Pi(p, N)$. The following two lemmas are easy to prove and characterize important properties of $\Pi(p, N)$ :

Lemma 9: Suppose that $p$ satisfies (21) and that $P$ in (7) is chosen as the positive definite solution to (22). We then have:

1) For any $N \geq 1$, we have $\Pi(0, N)=\phi_{1}^{T} P \phi_{1}$.

2) For any $0<p \leq 1 / \rho(A)^{2}$, we have

$$
\begin{aligned}
\Pi(p, 1) & =(1-p) \phi_{1}^{T} \Omega(p) \phi_{1} \\
\Pi(p, N+1) & =\Pi(p, N)-(1-p) p^{N}\left(\phi_{1}^{N}\right)^{T} \mathcal{S}(p) \phi_{1}^{N},
\end{aligned}
$$

where

$$
\mathcal{S}(p) \triangleq A^{T} \Omega(p) A-\phi_{1}^{T} \Omega(p) \phi_{1} .
$$

Proof: With $P$ as in (22), it holds that $\phi_{i}=\left(\phi_{1}\right)^{i}$, for all $i \geq 1$. The statements now follow directly from the definition of $\Pi(p, N)$, see (40).

Lemma 10: Suppose that $A \in \mathbb{R}$, that $p$ satisfies (21) and that $P$ in (7) is chosen as the positive definite solution to (22). We then have:

$$
\begin{gathered}
\mathcal{S}(p)=A^{2}\left(1-\left(\frac{\lambda}{B_{1}^{2} P+\lambda}\right)^{2}\right) \Omega(p) \geq 0, \\
P_{0}-\lim _{N \rightarrow \infty} \Pi(p, N)=\left(\frac{1-\phi_{1}^{2}}{1-p \phi_{1}^{2}}\right) P \geq 0 .
\end{gathered}
$$

Proof: With $A \in \mathbb{R}$ and $P$ as in (22), direct calculations give that

$$
\begin{aligned}
\phi_{i} & =A^{i}\left(\lambda /\left(B_{1}^{2}+P\right)\right)^{i}, \quad \forall i \geq 1, \\
\Omega(p) & =P /\left(1-p A^{2}\right), \quad \forall p \in\left(0,1 / A^{2}\right] .
\end{aligned}
$$

Expression (44) then follows directly from (43), whereas (45) can be proven by realizing that (40) is a geometric series.

As a consequence of Statement 1) in Lemma 9 and Theorem 3, we have that the NCS with quantization is MSS if there are no dropouts (and provided $\sigma_{n}^{2}<\infty$ ). On the other hand, Statement 2) in Lemma 9 gives that if $\mathcal{S}(p) \succeq 0$, then choosing
TABLE I

DROPOUT BOUNDS $p^{\sharp}$ WHICH ENSURE MSS AND AWSS OF (46) WHEN QUANTIZED PPC WITH HORIZON $N$ IS USED.

\begin{tabular}{|c|c|c|c|c|c|c|}
\hline$N$ & 1 & 2 & 3 & 4 & 5 & 6 \\
\hline$p^{\sharp}$ & $\begin{array}{c}4.883 \\
\times 10^{-5}\end{array}$ & $\begin{array}{c}8.823 \\
\times 10^{-3}\end{array}$ & $\begin{array}{c}8.793 \\
\times 10^{-2}\end{array}$ & $\begin{array}{c}1.512 \\
\times 10^{-1}\end{array}$ & $\begin{array}{c}1.626 \\
\times 10^{-1}\end{array}$ & $\begin{array}{c}1.628 \\
\times 10^{-1}\end{array}$ \\
\hline
\end{tabular}

larger horizons will make it easier to satisfy (42) and hence guarantee MSS and AWSS of the NCS. The beneficial effect of choosing large horizons is certainly not surprising since, as described in Section II-B, the buffering mechanism is aimed at achieving robustness with respect to packet dropouts.

Lemma 10 shows that for scalar plants, it always holds that $\mathcal{S}(p) \succeq 0$ and that if horizons are chosen large enough, then the bound on dropout-rates in (21) can be achieved with the packetized control method studied. As documented in Section IV-D below, these properties will often also be satisfied with higher order plants.

Remark 5: A key difference between Theorem 3 and the results of [20] is that in the latter work (as in other works, e.g., [9]) we assumed that the maximum number of consecutive packet dropouts was bounded. This allowed us to adopt in [20] a deterministic notion of stability, namely input-to-state stability (ISS). On the contrary, in the present setting, packet dropouts are assumed i.i.d., so that the maximum number of consecutive packet dropouts becomes unbounded. This makes deterministic stability notions unsuitable and the results of [20] are not directly applicable here.

\section{Numerical Example}

To illustrate our stability results, we consider a system of the form (1) where $B_{1}=B_{2}=\left[\begin{array}{lllll}1 & 1 & 1 & 1 & 1\end{array}\right]^{T}$ and where the matrix $A$ is randomly chosen:

$A=\left[\begin{array}{ccccc}-0.1331 & -0.5412 & -0.0008 & -1.0290 & -1.1746 \\ -1.2705 & -1.3335 & -0.2494 & 0.2431 & -1.0211 \\ -1.6636 & 1.0727 & 0.3966 & -1.2566 & -0.4017 \\ -0.7036 & -0.7121 & -0.2640 & -0.3472 & 0.1737 \\ 0.2809 & -0.0113 & -1.6640 & -0.9414 & -0.1161\end{array}\right]$.

We synthesize Quantized PPCs with different horizon lengths. All PPCs are nominally stabilizing and are designed according to (22), with $Q=I$, and $\lambda=1 / 20$.

The system in (46) is open-loop unstable, with $\rho(A)=$ 2.4786. Lemma 1 gives that a necessary condition for MSS of the closed loop system is $p \leq 0.1628$. Unfortunately, due to numerical issues, we could not use Lemma 3 to conclude upon necessary and sufficient conditions for MSS and AWSS of this system. In fact, for horizon lengths $N>2$ the conditioning number of $I-\mathcal{U} \otimes \mathcal{U}$ is larger than $10^{5}$. On the other hand, Theorem 3 allows us to conclude that if the dropout-rate $p$ is smaller than the bounds shown in Table I, then the NCS is MSS and AWSS. We can see that, despite the fact that the PPC does not require knowledge of $p$, the necessary condition for MSS (21) is achieved by the PPCs if the horizon length is chosen sufficiently large. 


\section{Closed Loop Performance}

Having studied AWSS and MSS of the NCS, we will next pursue our analysis further and tackle performance issues. For that purpose, we will first represent the NCS with dropouts, quantization and disturbances in the spectral domain. We will then investigate the effect of bit- and dropout-rates on the variance of the control error.

\section{A. Spectral Domain Representation}

To characterize spectral densities of the closed loop signals, we recall (26) and (27) and introduce the MIMO transfer functions:

$$
\begin{aligned}
& \mathcal{F}_{w}(z) \triangleq(z I-\mathcal{A}(p))^{-1} \mathcal{B}_{w}, \\
& \mathcal{F}_{n}(z) \triangleq(z I-\mathcal{A}(p))^{-1} \mathcal{B}_{n}(p) .
\end{aligned}
$$

As shown in Theorem 4 given below, $\mathcal{F}_{w}(z)$ and $\mathcal{F}_{n}(z)$ play a fundamental role for characterizing performance of the NCS.

Theorem 4: If the MJLS (15) is MSS and AWSS, then the spectral density of $\left\{\theta_{k}\right\}$ is given by:

$S_{\theta}\left(e^{j \omega}\right)=\mathcal{F}_{w}\left(e^{j \omega}\right) S_{w}^{H}\left(e^{j \omega}\right) \mathcal{F}_{w}^{H}\left(e^{j \omega}\right)+\frac{\sigma_{n}^{2}}{N} \mathcal{F}_{n}\left(e^{j \omega}\right) \mathcal{F}_{n}^{H}\left(e^{j \omega}\right)$.

Proof: See Appendix D.

The above result allows us to quantify the performance of the packetized NCS in Fig. 1 in the presence of quantization, random data dropouts and disturbances. In particular, (5) gives

$$
S_{\theta}\left(e^{j \omega}\right)=\left[\begin{array}{cc}
S_{x}\left(e^{j \omega}\right) & S_{x b}\left(e^{j \omega}\right) \\
S_{b x}\left(e^{j \omega}\right) & S_{b}\left(e^{j \omega}\right)
\end{array}\right]
$$

so that the spectral densities of $\left\{x_{k}\right\}$ and of $\left\{u_{k}\right\}$ are:

$$
\begin{aligned}
& S_{x}\left(e^{j \omega}\right)=\left[\begin{array}{ll}
I & 0
\end{array}\right] S_{\theta}\left(e^{j \omega}\right)\left[\begin{array}{l}
I \\
0
\end{array}\right], \\
& S_{u}\left(e^{j \omega}\right)=\left[\begin{array}{ll}
0 & e_{1}^{T}
\end{array}\right] S_{\theta}\left(e^{j \omega}\right)\left[\begin{array}{c}
0 \\
e_{1}
\end{array}\right] .
\end{aligned}
$$

Parseval's theorem then provides an explicit expression for the covariance matrix of $x$, namely:

$$
R_{x}(0)=\left[\begin{array}{ll}
I & 0
\end{array}\right]\left(\frac{\sigma_{n}^{2}}{N} \Xi_{n}(p)+\Xi_{w}(p)\right)\left[\begin{array}{l}
I \\
0
\end{array}\right],
$$

where $\Xi_{n}(p)$ solves (32), whereas

$$
\Xi_{w}(p) \triangleq \frac{1}{2 \pi} \int_{0}^{2 \pi} \mathcal{F}_{w}\left(e^{j \omega}\right) S_{w}^{H}\left(e^{j \omega}\right) \mathcal{F}_{w}^{H}\left(e^{j \omega}\right) d \omega
$$

Remark 6: Lemma 7 and Theorem 4 generalize results of [24], [50], [56]. To be more precise, whereas in the papers mentioned above only NCS's with a packet-dropping network carrying scalar signals are considered, the results in the present work are applicable to PPCs with $N \geq 1$, i.e., where sequences of plant inputs are transmitted.

\section{B. Rate-Distortion Analysis}

The quantization noise variance $\sigma_{n}^{2}$, see (14), is a user defined parameter, which affects bit-rate and achieved distortion. ECDQs use variable-length coding. We will study the expected length (in bits) of the codewords associated with the signal transmitted [27]. If the NCS is AWSS, then the expected bitrate satisfies

$$
R=\lim _{k \rightarrow \infty} \mathbb{E}\left\{v_{k}\right\}
$$

where the process $v_{k}$ represents the length (in bits) of the codewords associated with $q_{\mathcal{L}}\left(\xi_{k}+\eta_{k}\right)$, see (11).

As mentioned in Section II, we are interested in the socalled UDP-like case where there are no receipt acknowledgments. In order to guarantee that at the plant input side successful decoding of $q_{\mathcal{L}}\left(\xi_{k}+\eta_{k}\right)$, see (11), can be achieved despite random data-loss, we will use memoryless source coders. Thus, consecutive source vectors, $\xi_{k}+\eta_{k}$, are quantized independently.

In accordance with the control objective in (7), if the NCS is MSS (and thus AWSS, see Theorem 2), then we adopt the distortion measure $D$ given below as a figure of merit of the PPC quantization system:

$$
\begin{aligned}
D \triangleq \lim _{k \rightarrow \infty} \mathbb{E} & \left\{\left\|x_{k}\right\|_{Q}^{2}+\lambda\left(u_{k}\right)^{2}\right\} \\
& =\operatorname{tr}\left(Q^{1 / 2} R_{x}(0) Q^{T / 2}\right)+\lambda R_{u}(0),
\end{aligned}
$$

where $Q=Q^{T / 2} Q^{1 / 2}$. The distortion $D$ quantifies loss of performance of the NCS due to quantization, disturbances, and packet dropouts. Interestingly, we have the following result:

Lemma 11: Suppose that Assumption 1 holds, that the NCS is AWSS and MSS. Furthermore, consider $\Xi_{n}(p)$ which solves (32), and $\Xi_{w}(p)$ defined in (51). Then $D$ satisfies:

$$
D=\alpha \sigma_{n}^{2}+\beta,
$$

where:

$$
\begin{array}{r}
\left.\alpha \triangleq \frac{1}{N} \operatorname{tr}\left(\begin{array}{ll}
Q^{1 / 2} & 0
\end{array}\right] \Xi_{n}(p)\left[\begin{array}{ll}
Q^{1 / 2} & 0
\end{array}\right]^{T}\right) \\
+\frac{\lambda}{N}\left[\begin{array}{ll}
0 & e_{1}^{T}
\end{array}\right] \Xi_{n}(p)\left[\begin{array}{ll}
0 & e_{1}^{T}
\end{array}\right]^{T}>0 \\
\beta \triangleq \operatorname{tr}\left(\left[\begin{array}{ll}
Q^{1 / 2} & 0
\end{array}\right] \Xi_{w}(p)\left[\begin{array}{ll}
Q^{1 / 2} & 0
\end{array}\right]^{T}\right) \\
+\lambda\left[\begin{array}{ll}
0 & e_{1}^{T}
\end{array}\right] \Xi_{w}(p)\left[\begin{array}{ll}
0 & e_{1}^{T}
\end{array}\right]^{T}>0 .
\end{array}
$$

Proof: Follows directly from (49)-(51) and Parseval's theorem.

As a consequence of Lemma 11, the minimal distortion level achievable is $\beta$. This quantity depends upon the plant, controller parameters and dropout-rates. To achieve a small distortion level, we require the quantizer to introduce noise with a small variance $\sigma_{n}^{2}$. Unfortunately, this necessitates the use of large bit-rates $R$. In what follows, we will further elucidate this rate-distortion trade-off governing the design of the VQ. For that purpose, the characterization of the spectral densities obtained in Theorem 4, which is summarized in the following corollary, is of fundamental importance.

Corollary 1: Suppose that Assumption 1 holds, that the NCS is AWSS and MSS, and define

$$
\mathcal{H}(z) \triangleq\left[\begin{array}{ll}
I & 0
\end{array}\right](z I-\mathcal{A}(p))^{-1}
$$




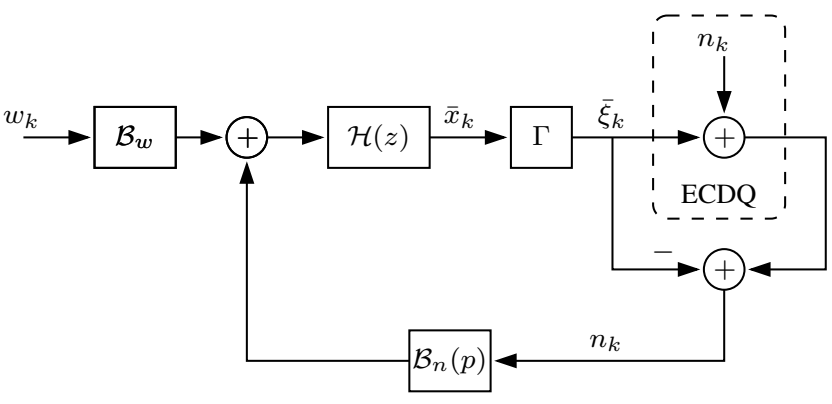

Fig. 2. Noise-Shaping Model of the NCS

Then the spectral density of $\bar{x}_{k}$ in the noise-shaping model in Fig. 2 is equal to the spectral density of the plant state $x_{k}$ in Fig. 1.

Proof: Follows directly from (9), (12), (48) and (49).

The equivalent noise-shaping structure provided by Fig. 2 allows us to analyze coding for the NCS by adapting techniques for LTI source coding systems.

Theorem 5 included below establishes an upper bound on the expected bit-rate of $\vec{u}_{k}$. Our result is valid at all rates and, thus, does not require high-rate approximations.

Theorem 5: Suppose that Assumption 1 holds, that the NCS is AWSS and MSS, and define the matrix

$$
\mathcal{Y} \triangleq\left[\begin{array}{ll}
\Gamma & 0
\end{array}\right]\left(\Xi_{n}(p)+(\alpha N /(D-\beta)) \Xi_{w}(p)\right)\left[\begin{array}{ll}
\Gamma & 0
\end{array}\right]^{T} .
$$

Then, for any $1 \leq N \in \mathbb{N}$, the bit-rate $R$ of $\vec{u}_{k}$ satisfies:

$$
\begin{aligned}
R(D) \leq & \frac{1}{2} \log _{2}(\operatorname{det}(I+\mathcal{Y}))+\frac{N}{2} \log _{2}\left(\frac{\pi e}{6}\right)+1 \\
= & \frac{1}{2} \sum_{i} \log _{2}\left(1+\nu_{i}\right)+\frac{N}{2} \log _{2}\left(\frac{\pi e}{6}\right)+1,
\end{aligned}
$$

where $\left\{\nu_{i}\right\}=\operatorname{eigs} \mathcal{Y}$.

Proof: See Appendix E.

Theorem 5 provides an analytical upper bound on expected bit-rates which guarantee a certain performance level when the PPC is used over channels with packet dropouts, when no acknowledgments are available.

We note that bounding the expected bit-rate $R$ will, in general, not guarantee that the instantaneous bit-rate is bounded. However, it can be shown that if $\xi_{k}$ has bounded support, then the cardinality of the codebook is finite [58] and, thus, also the instantaneous bit-rate is finite.

If we let $D \rightarrow \infty$, then the bound (57) becomes:

$$
\begin{aligned}
\lim _{D \rightarrow \infty} R(D) \leq \frac{1}{2} \log _{2} \operatorname{det}(I & \left.+\left[\begin{array}{ll}
\Gamma & 0
\end{array}\right] \Xi_{n}(p)\left[\begin{array}{ll}
\Gamma & 0
\end{array}\right]^{T}\right) \\
& +\frac{N}{2} \log _{2}\left(\frac{\pi e}{6}\right)+1 .
\end{aligned}
$$

It is interesting to note that the upper bound on the bit-rate is positively bounded away from zero, which is in agreement with the fact that, for stabilization, bit-rates cannot be made arbitrarily small; see, e.g., [2].

Conversely, if $\sigma_{n}^{2} \rightarrow 0$, so that quantization effects are negligible, then $R \rightarrow \infty$. In this case, the distortion $D \rightarrow \beta$ which depends only on the exogenous disturbance $w$, see (54).

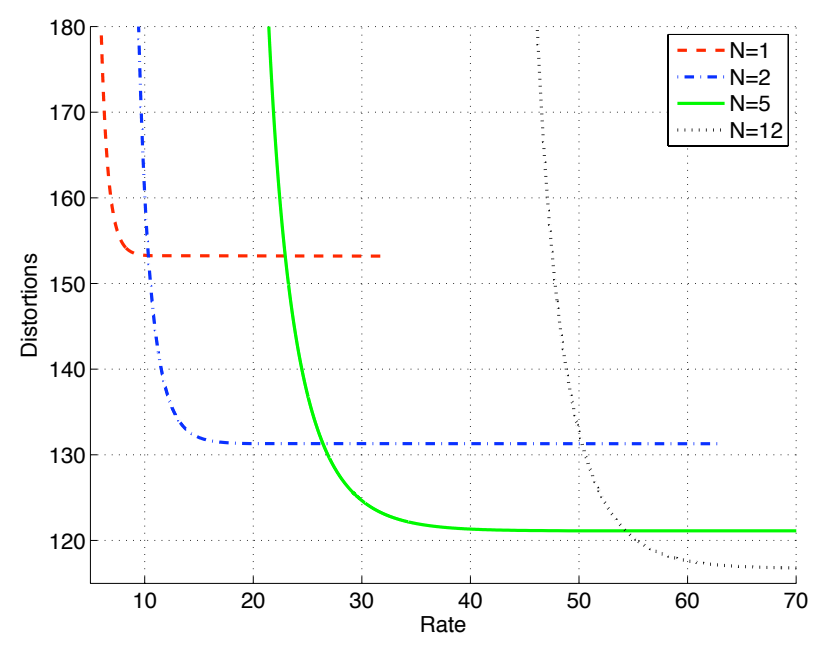

Fig. 3. Bound on $D(R)$ in (57) for different horizon lengths $N$, and for $p=0.05$. The asymptotics correspond to $\beta$ in (54) and the bound in (58).

Remark 7: Even in the simpler case of open-loop systems without dropouts, characterizing rate-distortion functions when limited delay is required, is a long standing open problem in source coding, formally known as causal source coding [59]. Thus, obtaining a complete characterization of distortion in closed-loop systems with bit-rate limitations is inherently difficult. Whilst achievable lower bounds on bit-rates which are required to guarantee MSS for NCS without losses are known [2], finding non-trivial lower bounds on bit-rates for performance remains an unsolved problem. Our results in Theorem 5 give upper-bounds on bit-rates which guarantee a certain performance level.

\section{Numerical Example}

To illustrate the performance bounds obtained, we use the example of Section IV-D. Fig. 3 shows the upper bound of the distortion $D$ as a function of $R$ which follows from (57). As can be seen in that figure, in the case examined, if a low distortion is desired and bit-rates are allowed to be moderate to high, then a larger horizon length should be chosen. However, at low bit-rates, shorter horizons may be preferable.

In Fig. 4 we examine the high-rate regime, in which case $D=\beta$, amounting to an unquantized system with packet dropouts. Again, choosing larger horizons leads to smaller distortions. It is worth recalling that, as seen in Section IV, the use of large horizons is also desirable from a stability point of view.

\section{Conclusions}

The present work has studied a control strategy for an architecture comprising an LTI plant with disturbances, and a bit-rate limited digital network affected by random packet dropouts. By using tools from stochastic control theory and Markov jump-linear systems, we have established results on stationarity and MSS of the resultant closed loop, when dropouts are Bernoulli distributed. It turns out that, if an entropy-coded dithered lattice vector quantizer is used, then the loop can be designed to be MSS for any bounded 


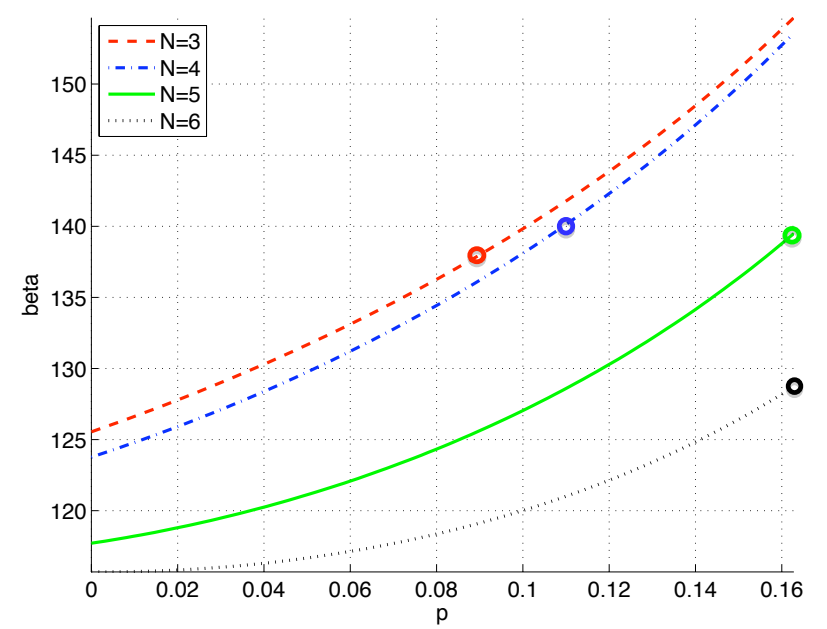

Fig. 4. High-rate analysis: $\beta$ as a function of dropout-rate $p$ for different horizon lengths $N$. The maximum dropout-rate examined is $p=1 / \rho(A)^{2}=$ 0.1628 . The circles correspond to the dropout-rates in Table I.

quantization noise variance. (For unstable plant models bitrates cannot be assigned arbitrarily.) Numerical results suggest that, if prediction horizons are chosen large enough, then MSS can be guaranteed provided dropout-rates are smaller than the inverse of the square of the spectral radius of the system matrix. The latter bound is a fundamental limit on stabilizability of networked control systems with dropouts. We have also characterized spectral densities of key signals in the loop and have developed an equivalent noise-shaping model. The spectral representation obtained has allowed us to bound the distortion as a function of disturbances, expected bit-rates, dropout-rates, and horizon-length. In general, better performance can be achieved if horizon-lengths and bit-rates are large and dropout-rates are small.

Future work includes adapting the method to use delayed packets, allowing for a network in the link from plant output to controller, and also investigating non-linear plant models. Preliminary results on the latter topic have been recently reported in [60].

\section{APPENDIX}

\section{A. Proof of Lemma 6}

Direct calculation shows that, for any $\Lambda$, it holds that:

$$
\begin{aligned}
(1-p) \bar{A}(0) \Lambda \bar{A}^{T}(0) & +p \bar{A}(1) \Lambda \bar{A}^{T}(1) \\
& =\mathcal{A}(p) \Lambda \mathcal{A}(p)^{T}+p(1-p) \widetilde{\mathcal{A}} \Lambda \widetilde{\mathcal{A}}^{T} .
\end{aligned}
$$

Thus, by (19) we have that the MJLS (15) is MSS and AWSS, iff there exists $\Lambda \succ 0$ :

$$
\mathcal{A}(p) \Lambda \mathcal{A}(p)^{T}-\Lambda+p(1-p) \widetilde{\mathcal{A}} \Lambda \widetilde{\mathcal{A}}^{T} \prec 0 .
$$

If in (29) we restrict $\Theta \succ 0$, then also $\Xi(p) \succ 0$. The result then follows directly from solving for $\Theta$ in (30), substituting into (29) and setting $\Lambda=\Xi(p)$.

In the semi-definite case $\Theta \succeq 0$, sufficiency of (29) can be proven following an idea akin to that used, e.g., in [24]. We first recall that $\mathcal{A}(p)$ is Schur, iff for some $\Omega \succ 0$ it holds that:

$$
\mathcal{A}(p) \Omega \mathcal{A}(p)^{T}-\Omega \prec 0 .
$$

We next set

$$
\Lambda=\Xi(p)+\delta \Omega \succ 0,
$$

where $\Omega$ is as in (60), $\Xi(p) \succeq 0$ is the solution to (30), and where $\delta>0$. Hence, by taking $\delta>0$ sufficiently small, Condition (29) implies that:

$$
p(1-p) \widetilde{\mathcal{A}} \Lambda \widetilde{\mathcal{A}}^{T}=p(1-p) \widetilde{\mathcal{A}}(\Xi(p)+\delta \Omega) \widetilde{\mathcal{A}}^{T} \prec \Theta .
$$

With $\Lambda$ as in (61) and by using (60) and (62), the left-hand side of (59) becomes:

$$
\begin{aligned}
\mathcal{A}(p)(\Xi(p)+\delta \Omega) \mathcal{A}(p)^{T}-(\Xi(p)+\delta \Omega) & \\
& +p(1-p) \widetilde{\mathcal{A}}(\Xi(p)+\delta \Omega) \widetilde{\mathcal{A}}^{T} \\
\prec & \mathcal{A}(p)(\Xi(p)+\delta \Omega) \mathcal{A}(p)^{T}-(\Xi(p)+\delta \Omega)+\Theta \\
= & \mathcal{A}(p) \Xi(p) \mathcal{A}(p)^{T}-\Xi(p)+\Theta+\delta\left(\mathcal{A}(p) \Omega \mathcal{A}(p)^{T}-\Omega\right) \\
= & \delta\left(\mathcal{A}(p) \Omega \mathcal{A}(p)^{T}-\Omega\right) \prec 0,
\end{aligned}
$$

which proves the result.

\section{B. Proof of Lemma 8}

It follows directly from (2) and (33), that

$$
\Delta \triangleq k_{1}-k_{0}
$$

is geometrically distributed according to

$$
\operatorname{Prob}(\Delta=i)=(1-p) p^{i-1}, \quad \forall i \in \mathbb{N} .
$$

Thus, the total probability formula gives:

$$
\begin{aligned}
\mathbb{E}_{\tilde{x}_{k_{0}}} V\left(\tilde{x}_{k_{1}}\right)=\mathbb{E}\left\{\mathbb{E}_{\tilde{x}_{k_{0}}}\left\{V\left(\tilde{x}_{k_{1}}\right) \mid \Delta\right\}\right\} \\
=(1-p) \sum_{i=1}^{\infty} p^{i-1} \mathbb{E}_{\tilde{x}_{k_{0}}}\left\{V\left(\tilde{x}_{k_{1}}\right) \mid \Delta=i\right\} \\
=(1-p) \sum_{i=1}^{N} p^{i-1} \mathbb{E}_{\tilde{x}_{k_{0}}}\left\{V\left(\tilde{x}_{k_{1}}\right) \mid \Delta=i\right\} \\
\quad+(1-p) \sum_{i=N+1}^{\infty} p^{i-1} \mathbb{E}_{\tilde{x}_{k_{0}}}\left\{V\left(\tilde{x}_{k_{1}}\right) \mid \Delta=i\right\} .
\end{aligned}
$$

If $\Delta \leq N$, then we have $\tilde{x}_{k_{1}}=\phi_{\Delta} \tilde{x}_{k_{0}}$ (see, e.g., [57]). On the other hand, since the packets transmitted have only $N$ elements and with the choice of $M$ made in (4), we have that if $\Delta>N$, then

$$
u_{\ell}=0, \quad \forall \ell \in\left\{k_{0}+N, k_{0}+N+1, \ldots, k_{1}-1\right\},
$$

so that, in this case,

$$
\tilde{x}_{k_{1}}=A^{k_{1}-k_{0}-N} \tilde{x}_{k_{0}+N}=A^{k_{1}-k_{0}-N} \phi_{N} \tilde{x}_{k_{0}} .
$$

Equations (35) and (64) then yield:

$$
\begin{aligned}
\mathbb{E}_{\tilde{x}_{k_{0}}} V\left(\tilde{x}_{k_{1}}\right)= & (1-p) \sum_{i=1}^{N} p^{i-1}\left\|\phi_{i} \tilde{x}_{k_{0}}\right\|_{P_{0}}^{2} \\
& +(1-p) \sum_{i=N+1}^{\infty} p^{i-1}\left\|A^{i-N} \phi_{N} \tilde{x}_{k_{0}}\right\|_{P_{0}}^{2} \\
= & (1-p) \sum_{i=1}^{N} p^{i-1}\left\|\phi_{i} \tilde{x}_{k_{0}}\right\|_{P_{0}}^{2} \\
& +(1-p) p^{N-1}\left(\left\|\phi_{N} \tilde{x}_{k_{0}}\right\|_{\Omega(p)}^{2}-\left\|\phi_{N} \tilde{x}_{k_{0}}\right\|_{P_{0}}^{2}\right),
\end{aligned}
$$

which gives (41). 


\section{Proof of Theorem 3}

We will first state, and prove, Corollary 2 and Lemma 12.

Corollary 2: System (15) is AWSS if and only if for all $d_{0} \in\{0,1\}$ and $\tilde{\theta}_{0}$ with bounded variance, it holds that

$$
\sum_{k=0}^{\infty} \mathbb{E}\left\{\left\|\tilde{x}_{k}\right\|^{2}\right\}<\infty
$$

Proof: [Corollary 2] By (17), we have that

$$
\sum_{k=0}^{\infty} \mathbb{E}\left\{\left\|\tilde{\theta}_{k}\right\|^{2}\right\}=\sum_{k=0}^{\infty} \mathbb{E}\left\{\left\|\tilde{x}_{k}\right\|^{2}+\left\|\tilde{b}_{k-1}\right\|^{2}\right\} .
$$

On the other hand, the buffering mechanism adopted allows one to bound:

$$
\begin{aligned}
\sum_{k=0}^{\infty}\left\|\tilde{b}_{k-1}\right\|^{2} & \leq N\left\|\tilde{b}_{-1}\right\|^{2}+N \sum_{k_{i} \in \mathcal{T}}\left\|\tilde{b}_{k_{i}}\right\|^{2} \\
& =N\left\|\tilde{b}_{-1}\right\|^{2}+N \sum_{k_{i} \in \mathcal{T}}\left\|\Psi^{-1} \Gamma \tilde{x}_{k_{i}}\right\|^{2} .
\end{aligned}
$$

The result now follows from noting that $\mathcal{T} \subseteq \mathbb{N}$ and applying Theorem 2.

Lemma 12: Suppose that $p$ satisfies (21) and consider (17). Then for all initial conditions $d_{0} \in\{0,1\}$ and $\tilde{\theta}_{0}$ with bounded variance there exist finite $C_{1}\left(\tilde{\theta}_{0}, d_{0}\right)$ and $C_{2}\left(\tilde{\theta}_{0}, d_{0}\right)$ such that

$$
\begin{aligned}
\mathbb{E}_{\left(\tilde{\theta}_{0}, d_{0}\right)}\left\{\sum_{\ell=0}^{k_{0}}\left\|\tilde{x}_{\ell}\right\|^{2}\right\} & \leq C_{1}\left(\tilde{\theta}_{0}, d_{0}\right) \\
\mathbb{E}_{\left(\tilde{\theta}_{0}, d_{0}\right)}\left\{V\left(\tilde{x}_{k_{0}}\right)\right\} & \leq C_{2}\left(\tilde{\theta}_{0}, d_{0}\right) .
\end{aligned}
$$

Proof: [Lemma 12] For $d_{0}=0$, then $k_{0}=0$ and (65) follows directly from the assumptions made and recalling that $V\left(\tilde{x}_{k_{0}}\right)=\left\|\tilde{x}_{k_{0}}\right\|_{P_{0}}^{2}$, see (35).

If $d_{0}=1$, then $k_{0}$ has geometric distribution

$$
\operatorname{Prob}\left(k_{0}=i\right)=(1-p) p^{i-1}, \quad \forall i \in \mathbb{N}, \quad \text { so that }
$$

$$
\begin{aligned}
& \mathbb{E}_{\left(\tilde{\theta}_{0}, d_{0}=1\right)}\left\{\sum_{\ell=0}^{k_{0}}\left\|\tilde{x}_{\ell}\right\|^{2}\right\} \\
& =(1-p) \sum_{i=1}^{N} p^{i-1} \mathbb{E}_{\left(\tilde{\theta}_{0}, d_{0}=1\right)}\left\{\sum_{\ell=0}^{k_{0}}\left\|\tilde{x}_{\ell}\right\|^{2} \mid k_{0}=i\right\} \\
& +(1-p) \sum_{i=N+1}^{\infty} p^{i-1} \mathbb{E}_{\left(\tilde{\theta}_{0}, d_{0}=1\right)}\left\{\sum_{\ell=0}^{N-1}\left\|\tilde{x}_{\ell}\right\|^{2} \mid k_{0}=i\right\} \\
& +(1-p) \sum_{i=N+1}^{\infty} p^{i-1} \mathbb{E}_{\left(\tilde{\theta}_{0}, d_{0}=1\right)}\left\{\sum_{\ell=N}^{k_{0}}\left\|\tilde{x}_{\ell}\right\|^{2} \mid k_{0}=i\right\} .
\end{aligned}
$$

The buffering mechanism employed and some algebraic manipulations then provide

$$
\begin{aligned}
& \mathbb{E}_{\left(\tilde{\theta}_{0}, d_{0}=1\right)}\left\{\sum_{\ell=0}^{k_{0}}\left\|\tilde{x}_{\ell}\right\|^{2}\right\} \\
& \leq(1-p) \sum_{i=1}^{\infty} p^{i-1} \mathbb{E}_{\left(\tilde{\theta}_{0}, d_{0}=1\right)}\left\{\sum_{\ell=0}^{N}\left\|\tilde{x}_{\ell}\right\|^{2} \mid k_{0} \geq N\right\} \\
& \quad+(1-p) \sum_{i=N+1}^{\infty} p^{i-1} \mathbb{E}_{\left(\tilde{\theta}_{0}, d_{0}=1\right)}\left\{\sum_{\ell=N}^{i}\left\|\tilde{x}_{\ell}\right\|^{2} \mid k_{0}=i\right\} \\
& =\mathbb{E}_{\left(\tilde{\theta}_{0}, d_{0}=1\right)}\left\{\sum_{\ell=0}^{N}\left\|\tilde{x}_{\ell}\right\|^{2} \mid k_{0} \geq N\right\} \\
& +(1-p) \sum_{i=N+1}^{\infty} p^{i-1} \mathbb{E}_{\left(\tilde{\theta}_{0}, d_{0}=1\right)}\left\{\sum_{\ell=N}^{i}\left\|A^{\ell-N} \tilde{x}_{N}\right\|^{2} \mid k_{0} \geq N\right\} \\
& =\mathbb{E}_{\left(\tilde{\theta}_{0}, d_{0}=1\right)}\left\{\sum_{\ell=0}^{N}\left\|\tilde{x}_{\ell}\right\|^{2} \mid k_{0} \geq N\right\} \\
& +p^{N} \mathbb{E}_{\left(\tilde{\theta}_{0}, d_{0}=1\right)}\left\{\left\|\tilde{x}_{N}\right\|^{2}+\sum_{j=0}^{\infty} p^{j}\left\|A^{j+1} \tilde{x}_{N}\right\|^{2} \mid k_{0} \geq N\right\} .
\end{aligned}
$$

If we now introduce $\Xi(p) \succ 0$ via the Lyapunov equation

$$
\Xi(p)=p A^{T} \Xi(p) A+I,
$$

then

$$
\sum_{j=0}^{\infty} p^{j}\left\|A^{j+1} \tilde{x}_{N}\right\|^{2}=\left\|A \tilde{x}_{N}\right\|_{\Xi(p)}^{2},
$$

so that (66) gives:

$$
\begin{aligned}
\mathbb{E}_{\left(\tilde{\theta}_{0}, d_{0}=1\right)}\{ & \left.\sum_{\ell=0}^{k_{0}}\left\|\tilde{x}_{\ell}\right\|^{2}\right\} \leq \mathbb{E}_{\left(\tilde{\theta}_{0}, d_{0}=1\right)}\left\{p^{N}\left\|\tilde{x}_{N}\right\|_{A^{T} \Xi(p) A+I}^{2}\right. \\
& \left.+\sum_{\ell=0}^{N}\left\|\tilde{x}_{\ell}\right\|^{2} \mid k_{0} \geq N\right\} \leq C_{1}\left(\tilde{\theta}_{0}, d_{0}=1\right),
\end{aligned}
$$

for some $C_{1}\left(\tilde{\theta}_{0}, d_{0}=1\right)<\infty$. The bound $C_{1}\left(\tilde{\theta}_{0}, d_{0}=1\right)$ is finite since, by assumption, the variances of $\tilde{x}_{0}$ and $\tilde{b}_{-1}$ are bounded and, given $k_{0} \geq N$, the plant inputs at instants $\ell \in\{0,1, \ldots, N-1\}$ are taken from $\tilde{b}_{-1}$. Existence of a finite bound $C_{2}\left(\tilde{\theta}_{0}, d_{0}=1\right)$ now follows from (35).

Proof: [Theorem 3] By Lemma 8, $V\left(\tilde{x}_{k_{i}}\right)$ constitutes a stochastic Lyapunov function for the NCS at the time instants $k_{i} \in \mathcal{T}$; see, e.g., [61]. In fact, since $V\left(\tilde{x}_{k_{0}}\right)=\left\|\tilde{x}_{k_{0}}\right\|_{P_{0}}^{2}$, Expression (42) gives that, for any given $\tilde{x}_{k_{0}}$, there exists a fixed $\alpha \in(0,1]$, such that: ${ }^{8}$

$$
\begin{aligned}
& \mathbb{E}_{\tilde{x}_{k_{0}}}\left\{V\left(\tilde{x}_{k_{1}}\right)\right\}-V\left(\tilde{x}_{k_{0}}\right)=\tilde{x}_{k_{0}}^{T}\left(\Pi(p, N)-P_{0}\right) \tilde{x}_{k_{0}} \\
& \leq-\alpha V\left(\tilde{x}_{k_{0}}\right) \text {. }
\end{aligned}
$$

Since $\left\{\tilde{x}_{k_{i}}\right\}_{k_{i} \in \mathcal{T}}$ is a Markov chain, we can use Theorem 2 of [61, Ch. 8.4.2], which gives:

$$
\mathbb{E}_{\tilde{x}_{k_{0}}}\left\{V\left(\tilde{x}_{k_{i}}\right)\right\} \leq(1-\alpha)^{i} V\left(\tilde{x}_{k_{0}}\right), \quad \forall i \geq 1 .
$$


To complete the proof, we use Corollary 2 and examine instants $k \notin \mathcal{T}, k>k_{0}$. Here we condition upon $\Delta$ defined in (63) and proceed along the same lines as in the proof of Lemma 12 to obtain:

$$
\begin{aligned}
& \mathbb{E}_{\tilde{x}_{k_{0}}}\left\{\sum_{\ell=k_{0}}^{k_{1}-1}\left\|\tilde{x}_{\ell}\right\|^{2}\right\} \\
& \leq(1-p) \sum_{i=1}^{\infty} p^{i-1} \mathbb{E}_{\tilde{x}_{k_{0}}}\left\{\sum_{\ell=k_{0}}^{k_{0}+N-1}\left\|\tilde{x}_{\ell}\right\|^{2} \mid \Delta \geq N\right\} \\
& \quad+(1-p) \sum_{i=N+1}^{\infty} p^{i-1} \mathbb{E}_{\tilde{x}_{k_{0}}}\left\{\sum_{\ell=k_{0}+N}^{k_{1}-1}\left\|\tilde{x}_{\ell}\right\|^{2} \mid \Delta=i\right\} \\
& =\mathbb{E}_{\tilde{x}_{k_{0}}}\left\{p^{N}\left\|\tilde{x}_{k_{0}+N}\right\|_{\Xi(p)}^{2}+\sum_{\ell=k_{0}}^{k_{0}+N-1}\left\|\tilde{x}_{\ell}\right\|^{2} \mid \Delta \geq N\right\},
\end{aligned}
$$

where $\Xi(p) \succ 0$ is as in (67). Thus, there exists $\gamma \in(0, \infty)$, such that

$$
\begin{aligned}
& \mathbb{E}_{\tilde{x}_{k_{0}}}\left\{\sum_{\ell=k_{0}}^{k_{1}-1}\left\|\tilde{x}_{\ell}\right\|^{2}\right\} \leq \gamma \mathbb{E}_{\tilde{x}_{k_{0}}}\left\{\left\|\tilde{x}_{k_{0}+N}\right\|_{P}^{2}\right. \\
& \left.+\sum_{\ell=k_{0}}^{k_{0}+N-1}\left(\left\|\tilde{x}_{\ell}\right\|_{Q}^{2}+\lambda\left(u_{\ell}\right)^{2}\right) \mid \Delta \geq N\right\}=\gamma \mathbb{E}_{\tilde{x}_{k_{0}}}\left\{V\left(\tilde{x}_{k_{0}}\right)\right\},
\end{aligned}
$$

In a similar way, it can be shown that

$$
\mathbb{E}_{\tilde{x}_{k_{i}}}\left\{\sum_{\ell=k_{i}}^{k_{i+1}-1}\left\|\tilde{x}_{\ell}\right\|^{2}\right\} \leq \gamma \mathbb{E}_{\tilde{x}_{k_{i}}}\left\{V\left(\tilde{x}_{k_{i}}\right)\right\}, \quad \forall k_{i} \in \mathcal{T}
$$

so that (68) gives:

$$
\begin{aligned}
\mathbb{E}_{\tilde{x}_{k_{0}}}\left\{\sum_{\ell=k_{i}}^{k_{i+1}-1}\left\|\tilde{x}_{\ell}\right\|^{2}\right\} & \leq \gamma \mathbb{E}_{\tilde{x}_{k_{0}}}\left\{V\left(\tilde{x}_{k_{i}}\right)\right\} \\
& \leq \gamma(1-\alpha)^{i} V\left(\tilde{x}_{k_{0}}\right), \quad \forall i \geq 1 .
\end{aligned}
$$

We can now use Lemma 12 to bound:

$$
\begin{aligned}
& \mathbb{E}_{\left(\tilde{\theta}_{0}, d_{0}\right)}\left\{\sum_{\ell=0}^{k_{m+1}-1}\left\|\tilde{x}_{\ell}\right\|^{2}\right\} \\
& \leq C_{1}\left(\tilde{\theta}_{0}, d_{0}\right)+\gamma \mathbb{E}_{\left(\tilde{\theta}_{0}, d_{0}\right)}\left\{\sum_{i=0}^{m} V\left(\tilde{x}_{k_{i}}\right)\right\} \\
& \leq C_{1}\left(\tilde{\theta}_{0}, d_{0}\right)+\gamma \frac{1-(1-\alpha)^{m}}{\alpha} \mathbb{E}_{\left(\tilde{\theta}_{0}, d_{0}\right)}\left\{V\left(\tilde{x}_{k_{0}}\right)\right\} \\
& =C_{1}\left(\tilde{\theta}_{0}, d_{0}\right)+\gamma \frac{1-(1-\alpha)^{m}}{\alpha} C_{2}\left(\tilde{\theta}_{0}, d_{0}\right), \quad \forall m \in \mathbb{N},
\end{aligned}
$$

expression, which upon taking the limit $m \rightarrow \infty$, provides:

$$
\mathbb{E}_{\left(\tilde{\theta}_{0}, d_{0}\right)}\left\{\sum_{\ell=0}^{\infty}\left\|\tilde{x}_{\ell}\right\|^{2}\right\} \leq C_{1}\left(\tilde{\theta}_{0}, d_{0}\right)+\frac{\gamma C_{2}\left(\tilde{\theta}_{0}, d_{0}\right)}{\alpha}<\infty .
$$

MSS and AWSS then follows from using the law of total expectation and Corollary 2.

\section{Proof of Theorem 4}

By conditioning upon the dropout process $d_{k}$, Equation (15) provides that the cross-covariance function of the processes $\nu_{k}$ and $\theta_{k}$ is given by: ${ }^{9}$

$$
\begin{aligned}
R_{\nu \theta}(\ell)= & \mathbb{E}\left\{\nu_{k+\ell} \theta_{k}^{T}\right\}=p \mathbb{E}\left\{\nu_{k+\ell}\left(\bar{A}(1) \theta_{k-1}+\bar{B}(1) \nu_{k-1}\right)^{T}\right\} \\
& +(1-p) \mathbb{E}\left\{\nu_{k+\ell}\left(\bar{A}(0) \theta_{k-1}+\bar{B}(0) \nu_{k-1}\right)^{T}\right\} \\
= & \mathbb{E}\left\{\nu_{k+\ell} \theta_{k-1}^{T}\right\} \mathcal{A}(p)^{T}+\mathbb{E}\left\{\nu_{k+\ell} \nu_{k-1}^{T}\right\} \mathcal{B}(p)^{T} \\
= & R_{\nu \theta}(\ell+1) \mathcal{A}(p)^{T}+R_{\nu}(\ell+1) \mathcal{B}(p)^{T},
\end{aligned}
$$

where $R_{\nu}(\ell)$ is the covariance function of $\nu_{k}$, and $\mathcal{A}(p)$ and $\mathcal{B}(p)$ are as in (25).

By taking two-sided $z$-Transform, we obtain that the crossspectrum satisfies:

$$
S_{\nu \theta}(z)=S_{\nu}(z) \mathcal{B}(p)^{T} z\left(I-z \mathcal{A}(p)^{T}\right)^{-1} .
$$

Similarly, the covariance function of $\theta_{k}$ is given by:

$$
\begin{aligned}
R_{\theta}(\ell)= & \mathbb{E}\left\{\theta_{k+\ell} \theta_{k}^{T}\right\}=p \mathbb{E}\left\{\theta_{k+\ell}\left(\bar{A}(1) \theta_{k-1}+\bar{B}(1) \nu_{k-1}\right)^{T}\right\} \\
& +(1-p) \mathbb{E}\left\{\theta_{k+\ell}\left(\bar{A}(0) \theta_{k-1}+\bar{B}(0) \nu_{k-1}\right)^{T}\right\} \\
= & \mathbb{E}\left\{\theta_{k+\ell} \theta_{k-1}^{T}\right\} \mathcal{A}(p)^{T}+\mathbb{E}\left\{\theta_{k+\ell} \nu_{k-1}^{T}\right\} \mathcal{B}(p)^{T} \\
= & R_{\theta}(\ell+1) \mathcal{A}(p)^{T}+R_{\theta \nu}(\ell+1) \mathcal{B}(p)^{T} .
\end{aligned}
$$

Since $R_{\theta \nu}(\ell)=R_{\nu \theta}^{T}(-\ell)$, we obtain that:

$$
\begin{aligned}
& S_{\theta}(z)=S_{\nu \theta}^{T}\left(z^{-1}\right) \mathcal{B}(p)^{T} z\left(I-z \mathcal{A}(p)^{T}\right)^{-1} \\
& \quad=(z I-\mathcal{A}(p))^{-1} \mathcal{B}(p) S_{\nu}^{T}\left(z^{-1}\right)\left(\left(z^{-1} I-\mathcal{A}(p)\right)^{-1} \mathcal{B}(p)\right)^{T}
\end{aligned}
$$

after replacing (69). On the other hand, the processes $w_{k}$ and $n_{k}$ are uncorrelated. It therefore holds that:

$$
\begin{array}{r}
\mathcal{B}(p) S_{\nu}^{T}\left(z^{-1}\right) \mathcal{B}(p)^{T}=\mathcal{B}(p)\left[\begin{array}{cc}
S_{w}^{T}\left(z^{-1}\right) & 0 \\
0 & S_{n}^{T}\left(z^{-1}\right)
\end{array}\right] \mathcal{B}(p)^{T} \\
=\mathcal{B}_{w} S_{w}^{T}\left(z^{-1}\right) \mathcal{B}_{w}^{T}+\mathcal{B}_{n}(p) S_{n}^{T}\left(z^{-1}\right) \mathcal{B}_{n}(p)^{T},
\end{array}
$$

where $\mathcal{B}_{n}(p)$ is as in (27). The result now follows from setting $z=e^{j \omega}$ and noting that, by (13), we have $S_{n}\left(e^{j \omega}\right)=\sigma_{n}^{2} / N$.

\section{E. Proof of Theorem 5}

By assumption, the loop is AWSS. Thus, results of [48] and [47] give that the coding rate of the ECDQ in Fig. 2 is related to the conditional entropy of the quantized signal, where conditioning is with respect to the dither signal as follows: ${ }^{10}$

$$
R \leq H\left(q_{\mathcal{L}}\left(\xi_{k}+\eta_{k}\right) \mid \eta_{k}\right)+1=I\left(\xi_{k} ; \xi_{k}+n_{k}\right)+1 .
$$

By following as in, e.g., [64, Lemma 2], one can show that $I\left(\xi_{k} ; \xi_{k}+n_{k}\right)$ can be upper bounded by replacing the variables in play with their Gaussian counterparts according to:

$$
I\left(\xi_{k} ; \xi_{k}+n_{k}\right) \leq I\left(\bar{\xi}_{k} ; \bar{\xi}_{k}+\bar{n}_{k}\right)+\mathcal{D}\left(n_{k} \| \bar{n}_{k}\right),
$$

${ }^{9}$ Note that all processes are zero-mean. Furthermore, since the MJLS is assumed MSS, by Lemma $4, \mathcal{A}(p)$ is Schur.

${ }^{10}$ Massey showed in [62] that an appropriate concept for studying minimum bit-rates in feedback systems is the directed mutual information. However, its achievability requires that the decoder have access to all previous transmissions. Thus results in [62], and also in [63] and other works, are not immediately applicable in the situation under study in the present work. 
where the processes $\bar{n}_{k}, \bar{w}_{k}$ and $\bar{\xi}_{k}$ are given by:

$$
\begin{gathered}
\bar{n}_{k} \sim \mathcal{N}\left(0,\left(\sigma_{n}^{2} / N\right) I\right), \quad \bar{w}_{k} \sim \mathcal{N}\left(0, R_{w}(0)\right), \\
\bar{\xi}_{k}=\left[\begin{array}{ll}
\Gamma & 0
\end{array}\right] \mathcal{F}_{w}(z) \bar{w}_{k}+\left[\begin{array}{ll}
\Gamma & 0
\end{array}\right] \mathcal{F}_{n}(z)\left(\bar{\xi}_{k}+\bar{n}_{k}\right) .
\end{gathered}
$$

The divergence in (71) describes a distance of quantization noise from Gaussianity. For ECDQs, it is bounded by [48]

$$
\mathcal{D}\left(n_{k} \| \bar{n}_{k}\right) \leq \frac{N}{2} \log _{2}(2 \pi e \mathcal{G})
$$

By definition, $I\left(\bar{\xi}_{k} ; \bar{\xi}_{k}+\bar{n}_{k}\right)=h\left(\bar{\xi}_{k}+\bar{n}_{k}\right)-h\left(\bar{\xi}_{k}+\bar{n}_{k} \mid \bar{\xi}_{k}\right)$, which can be simplified to

$$
\begin{aligned}
I\left(\bar{\xi}_{k} ; \bar{\xi}_{k}+\bar{n}_{k}\right)=h\left(\bar{\xi}_{k}+\bar{n}_{k}\right)-h\left(\bar{n}_{k}\right) \\
=\frac{1}{2} \log _{2}\left((2 \pi e)^{N} \operatorname{det}\left(R_{\xi}(0)+R_{n}(0)\right)\right) \\
\quad-\frac{1}{2} \log _{2}\left((2 \pi e)^{N} \operatorname{det}\left(R_{n}(0)\right)\right) \\
=\frac{1}{2} \log _{2}\left(\frac{\operatorname{det}\left(\left(\sigma_{n}^{2} / N\right) I+R_{\xi}(0)\right)}{\left(\sigma_{n}^{2} / N\right)^{N}}\right) \\
=\frac{1}{2} \log _{2}\left(\operatorname{det}\left(I+\left(N / \sigma_{n}^{2}\right) R_{\xi}(0)\right)\right),
\end{aligned}
$$

where we have used the fact that $\bar{\xi}_{k}$ and $\bar{n}_{k}$ are Gaussian and independent (recall Assumption 1). The inequality in (57) now follows from (70)-(73), the fact that $\mathcal{G} \leq 1 / 12$ for any lattice [46], by noting that $R_{\bar{\xi}}(0)=R_{\xi}(0)=\Gamma R_{x}(0) \Gamma^{T}$ and using (50) and (53). Finally, the right hand side equality in (57) can be easily verified.

\section{REFERENCES}

[1] J. P. Hespanha, P. Naghshtabrizi, and Y. Xu, "A survey of recent results in networked control systems," Proc. IEEE, vol. 1, no. 95, pp. 138-162, Jan. 2007.

[2] G. N. Nair, F. Fagnani, S. Zampieri, and R. J. Evans, "Feedback control under data rate constraints: An overview," Proc. IEEE, vol. 95, no. 1, pp. 108-137, Jan. 2007.

[3] L. Schenato, B. Sinopoli, M. Franceschetti, K. Poolla, and S. S. Sastry, "Foundations of control and estimation over lossy networks," Proc. IEEE, vol. 95, no. 1, pp. 163-187, Jan. 2007.

[4] A. S. Matveev and A. V. Savkin, Estimation and Control over Communication Networks. Birkäusser, 2009.

[5] J. R. Moyne and D. M. Tilbury, "The emergence of industrial control networks for manufacturing control, diagnostics, and safety data," Proc. IEEE, vol. 95, no. 1, pp. 29-47, Jan. 2007.

[6] D. Hristu-Varsakelis and W. S. Levine, Eds., Handbook of Networked and Embedded Systems. Boston, MA: Birkhäuser, 2005.

[7] S. Yüksel and T. Bassar, "Minimum rate coding for LTI systems over noiseless channels," IEEE Trans. Automat. Contr., vol. 51, no. 12, pp. 1878-1887, Dec. 2006.

[8] N. C. Martins, M. A. Dahleh, and N. Elia, "Feedback stabilization of uncertain systems in the presence of a direct link," IEEE Trans. Automat. Contr., vol. 51, no. 3, pp. 438-447, Mar. 2006.

[9] J. Xiong and J. Lam, "Stabilization of linear systems over networks with bounded packet loss," Automatica, vol. 43, pp. 80-87, 2007.

[10] N. Elia, "Remote stabilization over fading channels," Syst. \& Contr. Lett., pp. 237-249, 2005.

[11] A. Bemporad, "Predictive control of teleoperated constrained systems with unbounded communication delays," in Proc. IEEE Conf. Decis. Contr., Tampa, Florida, 1998, pp. 2133-2138.

[12] A. Casavola, E. Mosca, and M. Papini, "Predictive teleoperation of constrained dynamic systems via internet-like channels," IEEE Trans. Contr. Syst. Technol., vol. 14, no. 4, pp. 681-694, July 2006.

[13] P. L. Tang and C. W. de Silva, "Stability validation of a constrained model predictive networked control system with future input buffering," Int. J. Contr., vol. 80, no. 12, pp. 1954-1970, Dec. 2007.
[14] G. P. Liu, J. X. Mu, D. Rees, and S. C. Chai, "Design and stability analysis of networked control systems with random communication time delay using the modified MPC,' Int. J. Contr., vol. 79, no. 4, pp. 288297, Apr. 2006.

[15] Y.-B. Zhao, G. P. Liu, and D. Rees, "Improved predictive control approach to networked control systems," IET Control Theory Appl., vol. 2, no. 8, pp. 675-681, Aug. 2008.

[16] G. Pin and T. Parisini, "Stabilization of networked control systems by nonlinear model predictive control: A set invariance approach," in Nonlinear Model Predictive Control: Towards New Challenging Applications, ser. LNCIS, L. Magni, D. M. Raimondo, and F. Allgöwer, Eds. Berlin Heidelberg: Springer-Verlag, 2009, vol. 384, pp. 195-204.

[17] D. E. Quevedo, E. I. Silva, and G. C. Goodwin, "Packetized predictive control over erasure channels," in Proc. Amer. Contr. Conf., New York, N.Y., 2007.

[18] R. Findeisen and P. Varutti, "Stabilizing nonlinear predictive control over nondeterministic networks," in Nonlinear Model Predictive Control: Towards New Challenging Applications, ser. LNCIS, L. Magni, D. M. Raimondo, and F. Allgöwer, Eds. Berlin Heidelberg: Springer-Verlag, 2009, vol. 384, pp. 167-179.

[19] D. E. Quevedo, E. I. Silva, and G. C. Goodwin, "Control over unreliable networks affected by packet erasures and variable transmission delays," IEEE J. Select. Areas Commun., vol. 26, no. 4, pp. 672-685, May 2008.

[20] D. E. Quevedo and D. Nešić, "Input-to-state stability of packetized predictive control over unreliable networks affected by packet-dropouts," IEEE Trans. Automat. Contr., 2010, to appear.

[21] V. Gupta, B. Sinopoli, S. Adlakha, and A. Goldsmith, "Receding horizon networked control," in Proc. Allerton Conf. Communications, Control, and Computing, Monticello, IL, 2006.

[22] L. Schenato, "To zero or to hold control inputs with lossy links?" IEEE Trans. Automat. Contr., vol. 54, no. 5, pp. 1093-1099, May 2009.

[23] O. C. Imer, S. Yüksel, and T. Bassar, "Optimal control of LTI systems over unreliable communication links," Automatica, vol. 42, no. 9, pp. 1429-1439, Sept. 2006.

[24] Q. Ling and M. D. Lemmon, "Power spectral analysis of networked control systems with data dropouts," IEEE Trans. Automat. Contr., vol. 49, no. 6, pp. 955-960, June 2004.

[25] O. L. V. Costa, M. D. Fragoso, and R. P. Marques, Discrete-Time Markov Jump Linear Systems. London, U.K.: Springer-Verlag, 2005.

[26] Y. Ji, H. J. Chizeck, X. Feng, and K. A. Loparo, "Stability and control of discrete-time jump linear systems," Control Theory Adv. Technol., vol. 7, no. 2, pp. 247-270, 1991.

[27] T. M. Cover and J. A. Thomas, Elements of Information Theory, 2nd ed. Wiley-Interscience, 2006.

[28] T. Söderström, Discrete-Time Stochastic Systems. Prentice Hall, 1994.

[29] Y. Fang and K. A. Loparo, "Stochastic stability of jump linear systems," IEEE Trans. Automat. Contr., vol. 47, no. 7, pp. 1204-1208, July 2002.

[30] P. Seiler and R. Sengupta, "An $\mathcal{H}_{\infty}$ approach to networked control," IEEE Trans. Automat. Contr., vol. 50, no. 3, pp. 356-364, Mar. 2005.

[31] L. Xie and L. Xie, "Stability analysis of networked sampled-data linear systems with Markovian packet losses," IEEE Trans. Automat. Contr., vol. 54, no. 6, pp. 1375-1381, June 2009.

[32] S. Hu and W.-Y. Yan, "Stability robustness of networked control systems with respect to packet loss," Automatica, vol. 43, no. 7, pp. 1243-1248, July 2007.

[33] M. Sahebsara, T. Chen, and S. L. Shah, "Optimal $\mathcal{H}_{\infty}$ filtering in networked control systems with multiple packet dropouts," Syst. \& Contr. Lett., vol. 57, no. 9, pp. 692-702, Sept. 2008.

[34] O. L. V. Costa and E. F. Tuesta, "Finite horizon quadratic optimal control and a separation principle for Markovian jump linear systems," IEEE Trans. Automat. Contr., vol. 48, no. 10, pp. 1836-1842, Oct. 2003.

[35] B.-G. Park and W. H. Kwon, "Robust one-step receding horizon control of discrete-time Markovian jump uncertain systems," Automatica, vol. 38, pp. 1229-1235, 2002.

[36] J. C. Geromel, A. P. C. Gonçalves, and A. R. Fioravanti, "Dynamic output feedback control of discrete-time Markov jump linear systems through linear matrix inequalities," SIAM Journal on Control and Optimization, vol. 48, no. 2, pp. 573-593, 2009.

[37] O. L. V. Costa, E. O. Assumpção, E. K. Boukas, and R. P. Marques, "Constrained quadratic state feedback control of discrete-time Markovian jump linear systems," Automatica, vol. 35, no. 4, pp. 617-626, Apr. 1999.

[38] J. B. R. Do Val and T. Basar, "Receding horizon control of jump linear systems and a macroeconomic policy problem," Journal of Economic Dynamics \& Control, vol. 23, pp. 1099-1131, 1999. 
[39] A. N. Vargas, J. B. R. Do Val, and E. F. Costa, "Optimality conditions for the receding horizon control of Markov jump linear systems with non-observed chain and linear feedback controls," in Proc. IEEE Conf. Decis. Contr. and Europ. Contr. Conf., Seville, Spain, 2005, pp. 7308-7313.

[40] A. N. Vargas, W. Furloni, and J. B. R. Do Val, "Constrained model predictive control of jump linear systems with noise and non-observed Markov state," in Proc. IEEE Conf. Decis. Contr., 2006, pp. 929-934.

[41] D. E. Quevedo, G. C. Goodwin, and J. A. De Doná, "Finite constraint set receding horizon quadratic control," Int. J. Robust Nonlin. Contr., vol. 14, no. 4, pp. 355-377, Mar. 2004.

[42] D. Q. Mayne, J. B. Rawlings, C. V. Rao, and P. O. M. Scokaert, "Constrained model predictive control: Optimality and stability," Automatica, vol. 36, no. 6, pp. 789-814, June 2000.

[43] A. Gersho and R. M. Gray, Vector Quantization and Signal Compression. Boston, MA: Kluwer Academic, 1992.

[44] E. Agrell, T. Eriksson, A. Vardy, and K. Zeger, "Closest point search in lattices," IEEE Trans. Inform. Theory, vol. 48, no. 8, pp. 2201-2214, Aug. 2002.

[45] A. S. Matveev and A. V. Savkin, "The problem of stabilization of networked systems under computational power constraints," European J. Contr., vol. 15, no. 3-4, pp. 449-467, May-Aug. 2009.

[46] J. H. Conway and N. J. A. Sloane, Sphere packings, lattices and groups. London, U.K.: Springer-Verlag, 1988.

[47] R. Zamir and M. Feder, "On universal quantization by randomized uniform/lattice quantizers," IEEE Trans. Inform. Theory, vol. 38, no. 2, pp. 428-436, Mar. 1992.

[48] — " "On lattice quantization noise," IEEE Trans. Inform. Theory, vol. 42, no. 4, pp. 1152-1159, July 1996.

[49] R. Zamir, Y. Kochman, and U. Erez, "Achieving the Gaussian ratedistortion function by prediction," IEEE Trans. Inform. Theory, vol. 54, no. 7, pp. 3354-3364, July 2008.

[50] G. C. Goodwin, D. E. Quevedo, and E. I. Silva, "Architectures and coder design for networked control systems," Automatica, vol. 44, no. 1, pp. 248-257, Jan. 2008.

[51] M. S. Derpich, E. I. Silva, D. E. Quevedo, and G. C. Goodwin, "On optimal perfect reconstruction feedback quantizers," IEEE Trans. Signal Processing, vol. 56, no. 8, pp. 3871-3890, Aug. 2008.

[52] O. L. V. Costa and M. D. Fragoso, "Stability results for discrete-time linear systems with Markovian jumping parameters," J. Math. Anal. and Applicat., vol. 179, pp. 154-178, 1993.

[53] V. Gupta and N. C. Martins, "On stability in the presence of analog erasure channels between controller and actuator," IEEE Trans. Automat. Contr., vol. 55, no. 1, pp. 175-179, Jan. 2010.

[54] Y. Ji and H. J. Chizeck, "Jump linear quadratic Gaussian control: Steady state solution and testable conditions," Control Theory Adv. Technol., vol. 6, no. 3, pp. 289-319, 1990 .

[55] D. S. Bernstein, Matrix Mathematics, 2nd ed. Princeton, N.J.: Princeton University Press, 2009.

[56] E. I. Silva, G. C. Goodwin, and D. E. Quevedo, "On the design of control systems over unreliable channels," in Proc. Europ. Contr. Conf., Budapest, Hungary, 2009, pp. 377-382.

[57] D. P. Bertsekas, Dynamic Programming and Optimal Control, Vol.1. Belmont, MA.: Athena Scientific, 1995.

[58] A. György, T. Linder, P. A. Chou, and B. J. Betts, "Do optimal entropyconstrained quantizers have a finite or infinite number of codewords?" IEEE Trans. Inform. Theory, vol. 49, no. 11, pp. 3031-3037, Nov. 2003.

[59] D. Neuhoff and R. Gilbert, "Causal source codes," IEEE Trans. Inform. Theory, vol. 28, no. 5, pp. 701-713, Sept. 1982.

[60] D. E. Quevedo and D. Nešić, "On stochastic stability of packetized predictive control of non-linear systems over erasure channels," in 8th IFAC Symposium on Nonlinear Control Systems, September 2010.

[61] H. Kushner, Introduction to Stochastic Control. New York, N.Y.: Holt, Rinehart and Winston, Inc., 1971.

[62] J. L. Massey, "Causality, feedback and directed information," in Proc. IEEE Int. Symp. Inform. Theory, Waikiki, Hawaii, 1990, pp. 303 305.

[63] C. D. Charalambous and A. Farhadi, "LQG optimality and separation principle for general discrete time partially observed stochastic systems over finite capacity communication channels," Automatica, vol. 44, no. 12, pp. 3181-3188, Dec. 2008.

[64] M. S. Derpich, J. Østergaard, and G. C. Goodwin, "The quadratic Gaussian rate-distortion function for source uncorrelated distortions,' in Proc. Data Compression Conf., Snowbird, UT, 2008.

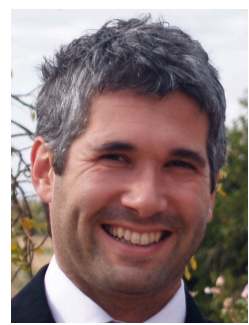

Daniel E. Quevedo (S'97-M'05) received Ingeniero Civil Electrónico and Magister en Ingeniería Electrónica degrees from the Universidad Técnica Federico Santa María, Valparaíso, Chile in 2000. In 2005, he received the Ph.D. degree from The University of Newcastle, Australia, where he is currently a research academic. He has been a visiting researcher at ETH Zürich, Switzerland, at Uppsala University, Sweden, at The University of Melbourne, Australia, at Aalborg University, Denmark, at Kyoto University, Japan, and at KTH Stockholm, Sweden.

Dr. Quevedo was supported by a full scholarship from the alumni association during his time at the Universidad Técnica Federico Santa María and received several university-wide prizes upon graduating. He received the IEEE Conference on Decision and Control Best Student Paper Award in 2003 and was also a finalist in 2002. In 2009, he was awarded an Australian Research Fellowship. His research interests cover several areas of automatic control, signal processing, communications, and power electronics.

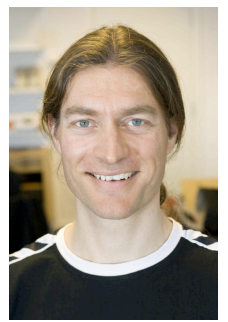

Jan Østergaard (S'98-M'99) received the M.Sc. degree in electrical engineering from Aalborg University, Aalborg, Denmark, in 1999 and the $\mathrm{Ph} . \mathrm{D}$. degree (cum laude) in electrical engineering from Delft University of Technology, Delft, The Netherlands, in 2007. From 1999 to 2002, he worked as an R\&D engineer at ETI A/S, Aalborg, Denmark, and from 2002 to 2003, he worked as an R\&D engineer at ETI Inc., Virginia, United States. Between September 2007 and June 2008, he worked as a post-doctoral researcher in the Centre for Complex Dynamic Systems and Control, School of Electrical Engineering and Computer Science, The University of Newcastle, NSW, Australia. He has also been a visiting researcher at Tel Aviv University, Tel Aviv, Israel, and at Universidad Técnica Federico Santa María, Valparaíso, Chile. He is currently a post-doctoral researcher at Aalborg University, Aalborg, Denmark.

Dr. Østergaard has received a Danish Independent Research Councils Young Researchers Award and a fellowship from the Danish Research Council for Technology and Production Sciences.

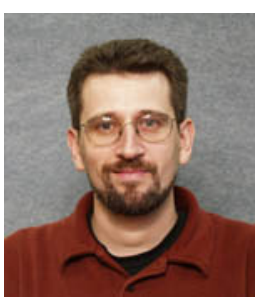

Dragan Nešić (SM'01-F'08) is a Professor in the Department of Electrical and Electronic Engineering (DEEE) at The University of Melbourne, Australia. He received his BE degree in Mechanical Engineering from The University of Belgrade, Yugoslavia in 1990, and his Ph.D. degree from Systems Engineering, RSISE, Australian National University, Canberra, Australia in 1997. Since February 1999 he has been with The University of Melbourne. His research interests include networked control systems, discrete-time, sampled-data and continuoustime nonlinear control systems, input-to-state stability, extremum seeking control, applications of symbolic computation in control theory, hybrid control systems, and so on. He was awarded a Humboldt Research Fellowship (2003) by the Alexander von Humboldt Foundation, an Australian Professorial Fellowship (2004-2009) and Future Fellowship (2010-2014) by the Australian Research Council.

$\mathrm{He}$ is a Fellow of IEEE and a Fellow of IEAust. He is currently a Distinguished Lecturer of CSS, IEEE (2008-2010). He is an Associate Editor for the journals Automatica, IEEE Transactions on Automatic Control, Systems and Control Letters and European Journal of Control. 